\title{
KRONECKER-BASED INFINITE LEVEL-DEPENDENT QBD PROCESSES
}

\author{
TUĞRUL DAYAR *** AND \\ M. CAN ORHAN, ${ }^{*}$ Bilkent University
}

\begin{abstract}
Markovian systems with multiple interacting subsystems under the influence of a control unit are considered. The state spaces of the subsystems are countably infinite, whereas that of the control unit is finite. A recent infinite level-dependent quasi-birth-and-death model for such systems is extended by facilitating the automatic representation and generation of the nonzero blocks in its underlying infinitesimal generator matrix with sums of Kronecker products. Experiments are performed on systems of stochastic chemical kinetics having two or more countably infinite state space subsystems. Results indicate that, even though more memory is consumed, there are many cases where a matrix-analytic solution coupled with Lyapunov theory yields a faster and more accurate steady-state measure compared to that obtained with simulation.
\end{abstract}

Keywords: Markov process; numerical analysis; simulation; biology; queues: theory

2010 Mathematics Subject Classification: Primary 60J22

Secondary 60J28; 92C $45 ; 92 \mathrm{D} 25$

\section{Introduction}

We consider Markovian systems with interacting subsystems under the influence of a control unit. The state space is countably infinite and $n$-dimensional with $n_{\mathrm{I}}$ countably infinite state variables and $n_{\mathrm{F}}$ finite state variables, where $n_{\mathrm{I}} \geq 2, n_{\mathrm{F}} \geq 0$, and $n=n_{\mathrm{I}}+n_{\mathrm{F}}$. Throughout the paper, we omit the word state and simply refer to the state variables as variables. The $n_{\mathrm{I}}$ countably infinite variables are used to represent the interacting $n_{\text {I }}$ subsystems and the $n_{\mathrm{F}}$ finite variables are associated with the control unit. When the control unit does not exist, we have $n=n_{\mathrm{I}}$. The state space of variable $i$ in the $n$-dimensional state space is denoted by $s_{i}$ and $s_{i} \subseteq \mathbb{Z}_{+}$for $i=1, \ldots, n$. Without loss of generality, let us assume that the first $n_{\mathrm{I}}$ indices are assigned to the interacting subsystems. Hence, $\wp_{i}$ is countably infinite for $i=1, \ldots, n_{\mathrm{I}}$ and finite for $i=n_{\mathrm{I}}+1, \ldots, n$.

We let $\delta$ be the state space of the underlying time-homogeneous, irreducible, continuoustime Markov chain (CTMC) [11], [30] corresponding to the system. Clearly, not all states in the product state space $X_{i=1}^{n} \delta_{i}$, where ' $X$ ' is the Cartesian product operator, are necessarily reachable. However, each state in $\delta$ is reachable from every other state in $\delta$. In many cases, $\delta$ is a proper subset of the product state space (i.e. $\delta \subset \mathrm{X}_{i=1}^{n} \delta_{i}$ ). In this paper we consider ergodic CTMCs associated with $\&$ and investigate the computation of their steady-state probability measures [11], [30].

Two classes of problems which can be modeled using the described $n$-dimensional state representation with $n_{\mathrm{I}}$ countably infinite variables come from stochastic chemical kinetics [14],

Received 15 March 2012; revision received 14 May 2012.

* Postal address: Department of Computer Engineering, Bilkent University, TR-06800 Bilkent, Ankara, Turkey.

** Email address: tugrul@cs.bilkent.edu.tr 
[19], [27], [33] and queueing networks [3], [11], [13], [15]. For the former, the $n_{\mathrm{I}}$ countably infinite variables represent the numbers of molecules of each chemical species existing in the system. For the latter, the $n_{\mathrm{I}}$ countably infinite variables represent the occupancies of queues with unbounded waiting space in the network. For both classes of problems, the remaining $n_{\mathrm{F}}$ finite variables in the $n$-dimensional state space can be perceived as forming the control unit. It is the former class of problems we consider in this paper and leave the investigation of the latter class of problems to another paper.

Recently, in [7] it has been shown that systems of stochastic chemical kinetics can be modeled as infinite level-dependent quasi-birth-and-death (LDQBD) processes. In particular, the maximum value among the $n_{\text {I }}$ countably infinite variables determines the level number, and the number of different possibilities for the $n_{\text {I }}$ countably infinite variables times the number of different possibilities for the $n_{\mathrm{F}}$ finite variables determines the number of states within a level. Assuming that the subset of states in $\delta$ corresponding to level $l$ is denoted by $\delta^{(l)}$, this yields a block tridiagonal infinitesimal generator matrix [2], [25]

$$
Q=\left(\begin{array}{cccccc}
Q_{0,0} & Q_{0,1} & & & & \\
Q_{1,0} & Q_{1,1} & Q_{1,2} & & & \\
& \ddots & \ddots & \ddots & & \\
& & Q_{l, l-1} & Q_{l, l} & Q_{l, l+1} & \\
& & & \ddots & \ddots & \ddots
\end{array}\right)
$$

in which the nonzero blocks at level $l$ are given by $Q_{l, l-1} \in \mathbb{R}_{\geq 0}^{\left|f^{(l)}\right| \times\left|f^{(l-1)}\right|}, Q_{l, l} \in \mathbb{R}^{\left|f^{(l)}\right| \times\left|f^{(l)}\right|}$, and $Q_{l, l+1} \in \mathbb{R}_{\geq 0}^{\left|f^{(l)}\right| \times\left|f^{(l+1)}\right|}$. These blocks are generally very sparse and their nonzero entries may have values depending on the level number. The off-diagonal entries of $Q$ are nonnegative, whereas its diagonal entries are negative. Level 0 forms the boundary and has two nonzero blocks. Clearly, the ordering of states within a level is only fixed up to a permutation. Observe that transitions are possible only between adjacent levels and the number of states within each level increases with increasing level number. The latter is due to the increase in the number of different possibilities for the $n_{\mathrm{I}}$ countably infinite variables according to the level definition being used.

We let $X(t)=\left(X_{1}(t), \ldots, X_{n}(t)\right)$ denote the state of the LDQBD process at time $t$, and let $\{X(t), t \geq 0\}$ be the associated CTMC process. Then the probability that the LDQBD process is in state $x \in \delta$ at time $t$ is given by $\operatorname{Pr}\{X(t)=x\}=\operatorname{Pr}\left\{X_{1}(t)=x_{1}, \ldots, X_{n}(t)=x_{n}\right\}$, and its steady-state probability distribution row vector, $\pi:=\lim _{t \rightarrow \infty} \operatorname{Pr}\{X(t)\}$, satisfies $\pi Q=0$ and $\sum_{x \in \delta} \pi(x)=1$ [11], [30]. When there is a pure birth process with rates upper bounding (or a birth-and-death process with birth and death rates respectively upper and lower bounding) those of the one-dimensional CTMC defined over levels, then relatively simple conditions related to nonexplosiveness [24] can be utilized to establish the LDQBD process's ergodicity. Alternatively, the Lyapunov function methods as discussed in [10] and [32] can be used. It is the latter approach we follow in this work since by using a judiciously chosen Lyapunov function, we can also obtain the values of lower and higher level numbers (called low and high, respectively) as in [7] between which a specified percentage of the steady-state probability mass lies when the LDQBD process is ergodic. Once these two level numbers are available, the computation of $\pi$ follows from a matrix-analytic method [12], [15], [20] proposed in [2]. In this method, first the conditional expected sojourn time matrix at level high, $R_{\mathrm{high}}$, is computed. Then, by using the recursive relationship

$$
R_{l}=Q_{l, l+1}\left(-Q_{l+1, l+1}-R_{l+1} Q_{l+2, l+1}\right)^{-1} \text { for } l \geq 0,
$$


the conditional expected sojourn time matrices between low and high are computed. After obtaining these matrices, steady-state probability subvectors for levels low to high are computed by the equation

$$
\pi^{(l+1)}=\pi^{(l)} R_{l} \quad \text { for } l \geq 0,
$$

where $\pi^{(l)}$ and $\pi^{(l+1)}$ are the steady-state probability subvectors of levels $l$ and $l+1$, respectively. The advantage of this approach compared to approximate methods (including simulation) lies in the fact that steady-state measures can be computed exactly up to machine precision by prescribing the difference between high and low sufficiently large.

The motivation for this study is based on the observation that, although it may be relatively easy to manually enumerate the states and generate the sparse nonzero blocks $Q_{l, l-1}, Q_{l, l}$, $Q_{l, l+1}$ within each level when $n_{\mathrm{I}}=2$, the task becomes unmanageable once $n_{\mathrm{I}}>2$. Hence, there is a need to be able to do this from the problem specification in an automated manner. We will see that this problem can be handled smoothly by introducing Kronecker products [3], [4], [22], [23] to cope with multidimensionality. Yet, an important requirement in this procedure is to be able to represent only the irreducible set of states associated with the underlying CTMC. Thanks to hierarchical Markovian models (HMMs) introduced in [3], this second problem can be solved without much difficulty as well. Armed with a Kronecker-based representation for infinite LDQBD processes, we finally undertake, possibly for the first time, a comparative study between stochastic simulation [9] and the matrix-analytic approach.

In the next section we introduce a specification for the class of problems we consider and build a Kronecker representation of the corresponding sparse nonzero blocks in $Q$. In the third section we provide a detailed example showing how this all works in practice. In the same section we also introduce three- and four-dimensional examples from the same domain. The fourth section outlines a technique for proving the finiteness of the set of states in which a specified percentage of the steady-state probability mass lies. Then we report on the results of numerical experiments with the matrix-analytic approach on the examples introduced earlier and with simulation. In the fifth section we conclude.

Throughout the paper, all vectors are column vectors except state vectors, consistent with the conventional definition of state probability vectors as row vectors. We represent by $e$ a column vector of $1 \mathrm{~s}$ and by $e_{i}$ the $i$ th column of the identity matrix, $I$. We denote by $\operatorname{diag}(a), \operatorname{subdiag}(a)$, and superdiag $(a)$ matrices with the entries of vector $a$ along their diagonal, subdiagonal, and superdiagonal, respectively. All other entries of these matrices are 0 . A subscript under $I$ is used to indicate its order. Similarly, the subscript $m \times n$ under a matrix indicates that the matrix is $(m \times n)$. The lengths of the vectors are determined by the context in which they are used and ' $\mathrm{T}$, stands for transposition.

\section{Kronecker representation}

We consider systems of stochastic chemical kinetics defined by a set of $J$ transition classes over $\&$, and let $x=\left(x_{1}, \ldots, x_{n}\right) \in \mathbb{Z}_{+}^{1 \times n}$ denote a state in $\&$.

Definition 1. A transition class $j \in\{1, \ldots, J\}$ is a pair $\left(\psi^{(j)} \prod_{i=1}^{n} h^{(j, i)}\left(x_{i}\right), v^{(j)}\right)$, where $\psi^{(j)} \in \mathbb{R}_{>0}, h^{(j, i)}\left(x_{i}\right): \wp_{i} \rightarrow \mathbb{R}_{\geq 0}$, and $v^{(j)} \in \mathbb{Z}^{1 \times n}$ are respectively its state-independent transition rate, its state-dependent transition rate for variable $i \in\{1, \ldots, n\}$, and its state change vector. The first element of the pair,

$$
\alpha_{j}(x):=\psi^{(j)} \prod_{i=1}^{n} h^{(j, i)}\left(x_{i}\right),
$$


specifies the transition rate from state $x \in \&$ to state $\left(x+v^{(j)}\right) \in \S$. The second element of the pair, $v^{(j)} \in \mathbb{Z}^{1 \times n}$, specifies the successor state of the transition, where $v_{i}^{(j)}$ denotes the change in variable $i$ due to transition class $j$.

The following definition associates $n_{\text {I }}$ transition rate matrices with each transition class in Definition 1.

Definition 2. The transition rate matrix of the countably infinite variable $i \in\left\{1, \ldots, n_{\mathrm{I}}\right\}$ for transition class $j \in\{1, \ldots, J\}$, denoted by $Z^{(j, i)} \in \mathbb{R}_{\geq 0}^{\left|f_{i}\right| \times\left|\delta_{i}\right|}$, is given entrywise as

$$
Z^{(j, i)}\left(x_{i}, y_{i}\right)=\left\{\begin{array}{ll}
h^{(j, i)}\left(x_{i}\right) & \text { if } y_{i}=x_{i}+v_{i}^{(j)}, \\
0 & \text { otherwise, }
\end{array} \quad \text { for } x_{i}, y_{i} \in s_{i} .\right.
$$

Note that in Definition 2, only countably infinite variables are considered. We also define transition rate matrices for finite variables. However, for each transition class, we prefer to define a combined transition rate matrix for all finite variables since we have observed that in practice $\left|\wp_{i}\right|$ for $i=n_{\mathrm{I}}+1, \ldots, n$ is very small. Now, let $\bar{\delta}$ denote the set of states which finite variables can take. Then, $\bar{s} \subseteq X_{i=n_{\mathrm{I}}+1}^{n} s_{i}$.

Definition 3. When $n>n_{\mathrm{I}}$, the combined transition rate matrix of finite variables for transition class $j \in\{1, \ldots, J\}$, denoted by $\bar{Z}^{(j)} \in \mathbb{R}_{\geq 0}^{|\bar{\gamma}| \times|\bar{\gamma}|}$, is given entrywise as

$$
\begin{aligned}
& \bar{Z}^{(j)}\left(\left(x_{n_{\mathrm{I}}+1}, \ldots, x_{n}\right),\left(y_{n_{\mathrm{I}}+1}, \ldots, y_{n}\right)\right) \\
& \quad= \begin{cases}\prod_{i=n_{\mathrm{I}}+1}^{n} h^{(j, i)}\left(x_{i}\right) & \text { if }\left(y_{n_{\mathrm{I}}+1}, \ldots, y_{n}\right)=\left(x_{n_{\mathrm{I}}+1}, \ldots, x_{n}\right)+\left(v_{n_{\mathrm{I}}+1}^{(j)}, \ldots, v_{n}^{(j)}\right), \\
0 & \text { otherwise, }\end{cases}
\end{aligned}
$$

for $\left(x_{n_{\mathrm{I}}+1}, \ldots, x_{n}\right),\left(y_{n_{\mathrm{I}}+1}, \ldots, y_{n}\right) \in \bar{\S}$. When $n=n_{\mathrm{I}}$, it is assumed that $|\bar{\S}|=1$ and $\bar{Z}^{(j)}=(1)$.

We are interested in obtaining a Kronecker representation for the nonzero blocks of $Q$ from the state-independent transition rates and the transition rate matrices of Definitions 2 and 3. To this end, let us start by formally defining $f^{(l)}$.

Definition 4. The subset of states corresponding to level $l \in \mathbb{Z}_{+}$is given by

$$
\delta^{(l)}=\left\{x \in \delta \mid \max \left(x_{1}, \ldots, x_{n_{\mathrm{I}}}\right)=l\right\},
$$

so that $\&=\bigcup_{l=0}^{\infty} 8^{(l)}$.

The maximum function is justified by observing that the maximum-valued variable among $x_{1}, \ldots, x_{n_{\mathrm{I}}}$ in any state $x \in \delta$ changes by at most one through any transition due to the particular form of the state change vectors $v^{(j)}$ in the transition classes for systems of stochastic chemical kinetics. Observe that $f^{(l)} \cap f^{(k)}=\varnothing$ holds for $l \neq k$, where $l, k \in \mathbb{Z}_{+}$.

For each level $l$, the values a variable can take depend on the values of other variables. Therefore, first we define a partition of the values a countably infinite variable can take where there is no such dependency in a way similar to HMMs in [3]. Then we introduce a partition of $f^{(l)}$ in Definition 4 based on the partitions of countably infinite variables defined before. 
Definition 5. Let

$$
\delta_{i}^{(l, u)}=\left\{\begin{array}{ll}
\left\{x_{i} \mid 0 \leq x_{i} \leq l-1\right\} & \text { if } i<u, \\
\{l\} & \text { if } i=u, \\
\left\{x_{i} \mid 0 \leq x_{i} \leq l\right\} & \text { if } i>u,
\end{array} \text { for } i, u \in\left\{1, \ldots, n_{\mathrm{I}}\right\} .\right.
$$

Then partition $u \in\left\{1, \ldots, n_{\mathrm{I}}\right\}$ of $s^{(l)}$, denoted by $s^{(l, u)}$, is given by

$$
s^{(l, u)}=\left\{x \in s^{(l)} \mid\left(x_{1}, \ldots, x_{n_{\mathrm{I}}}\right) \in \underset{i=1}{n_{\mathrm{I}}} s_{i}^{(l, u)} \text { and }\left(x_{n_{\mathrm{I}}+1}, \ldots, x_{n}\right) \in \bar{s}\right\},
$$

so that $f^{(l)}=\bigcup_{u=1}^{n_{\mathrm{I}}} f^{(l, u)}$. Without loss of generality, the partitions $f^{(l, u)}$ are assumed to be ordered within $f^{(l)}$ according to the increasing partition index, $u$.

Observe that $\delta^{(l, u)} \cap \delta^{(l, w)}=\varnothing$ for $u \neq w$, where $u, w \in\left\{1, \ldots, n_{\mathrm{I}}\right\}$ and $l>0$. For given level $l \in \mathbb{Z}_{+}$and noting that $n \geq n_{\mathrm{I}} \geq 2$, by Definition 5 we have

$$
\left|\S^{(l, u)}\right|=(l+1)^{n_{\mathrm{I}}-u}(l)^{u-1}|\bar{\S}| \quad \text { for } u \in\left\{1, \ldots, n_{\mathrm{I}}\right\} .
$$

Then the number of states in level $l$ can be obtained as

$$
\left|\mathcal{\delta}^{(l)}\right|=\sum_{u=1}^{n_{\mathrm{I}}}\left|\mathcal{\delta}^{(l, u)}\right|=|\bar{\delta}|\left((l+1)^{n_{\mathrm{I}}}-(l)^{n_{\mathrm{I}}}\right),
$$

which implies that the number of states at level $l \in \mathbb{Z}_{+}$is $O\left(l^{n_{\mathrm{I}}-1}\right)$.

Now, we are in a position to introduce the Kronecker representation of nonzero blocks in $Q$ following the partitions of the subset of states at each level given by Definition 5 .

Definition 6. The nonzero blocks $Q_{0,0}, Q_{0,1}, Q_{1,0}$, and $Q_{l, m}$ for $l>0$ and $m \in\{l-1, l, l+1\}$ are respectively $(1 \times 1),\left(1 \times n_{\mathrm{I}}\right),\left(n_{\mathrm{I}} \times 1\right)$, and $\left(n_{\mathrm{I}} \times n_{\mathrm{I}}\right)$ block matrices as in

$$
\begin{aligned}
& Q_{0,0}=\left(\begin{array}{lll}
Q_{0,0}^{(1,1)}
\end{array}\right), \quad Q_{0,1}=\left(\begin{array}{lll}
Q_{0,1}^{(1,1)} & \cdots & Q_{0,1}^{\left(1, n_{\mathrm{I}}\right)}
\end{array}\right), \\
& Q_{1,0}=\left(\begin{array}{c}
Q_{1,0}^{(1,1)} \\
\vdots \\
Q_{1,0}^{\left(n_{\mathrm{I}}, 1\right)}
\end{array}\right), \quad Q_{l, m}=\left(\begin{array}{ccc}
Q_{l, m}^{(1,1)} & \cdots & Q_{l, m}^{\left(1, n_{\mathrm{I}}\right)} \\
\vdots & \ddots & \vdots \\
Q_{l, m}^{\left(n_{\mathrm{I}}, 1\right)} & \cdots & Q_{l, m}^{\left(n_{\mathrm{I}}, n_{\mathrm{I}}\right)}
\end{array}\right) .
\end{aligned}
$$

Furthermore, the blocks of $Q_{l, m}$ can be written in terms of state-independent transition rates and transition rate matrices as in

$$
Q_{l, m}^{(u, w)}= \begin{cases}\tilde{Q}_{l, m}^{(u, w)}-\operatorname{diag}\left(\sum_{m^{\prime}=l-1}^{l+1} \sum_{w^{\prime}=1}^{n_{\mathrm{I}}} \tilde{Q}_{l, m^{\prime}}^{\left(u, w^{\prime}\right)} e\right) & \text { if } u=w \text { and } l=m, \\ \tilde{Q}_{l, m}^{(u, w)} & \text { otherwise }\end{cases}
$$

for $l, m, u, w \geq 0$, where

$$
\tilde{Q}_{l, m}^{(u, w)}=\sum_{j=1}^{J} \psi^{(j)}\left(\left(\bigotimes_{i=1}^{n_{\mathrm{I}}} Z^{(j, i)}\left(s_{i}^{(l, u)}, s_{i}^{(m, w)}\right)\right) \otimes \bar{Z}^{(j)}\right)
$$

and $Z^{(j, i)}\left(f_{i}^{(l, u)}, f_{i}^{(m, w)}\right)$ denotes the submatrix of $Z^{(j, i)}$ incident on row indices in $f_{i}^{(l, u)}$ and column indices in $f_{i}^{(m, w)}$. The first summation in diag should have a starting index of 0 rather 
than -1 for the equation of the block $Q_{0,0}^{(1,1)}$, and the second summation in diag should have an ending index of 1 rather than $n_{\mathrm{I}}$ for the equation of the blocks $Q_{1,1}^{(1,1)}, \ldots, Q_{1,1}^{\left(n_{\mathrm{I}}, n_{\mathrm{I}}\right)}$ when $m^{\prime}=l-1$.

In the next section we introduce four examples we will be using in the experiments. Due to space limitations, we provide the nonzero blocks of $Q$ and the Kronecker representation of their subblocks for one example only. To that end, we choose the three-dimensional example since it indicates how the approach applies in higher dimensions, yet its subblocks can still be written in a readable form. Kronecker representation of subblocks of nonzero blocks for all examples can be found in [21].

\section{Examples}

Example 1. (Gene expression.) Consider a system of stochastic chemical kinetics modeling the biological process associated with a gene expression [31]. The transition classes of this example are given in Table 1. Here, $n=n_{\mathrm{I}}=2, n_{\mathrm{F}}=0, x=\left(x_{1}, x_{2}\right), J=4$, and $\lambda, \mu, \delta_{1}$, $\delta_{2} \in \mathbb{R}_{>0}$. Hence, we have $\varsigma_{1}=\varsigma_{2}=\mathbb{Z}_{+},|\bar{\delta}|=1$, and $\delta=\wp_{1} \times \wp_{2}=\mathbb{Z}_{+}^{1 \times 2}$. Note that (2) implies $2 l+1$ states at level $l \in \mathbb{Z}_{+}$.

The transition rate matrices of the model from Table 1 and Definition 2 are obtained as

$$
\begin{array}{ll}
Z^{(1,2)}=Z^{(3,2)}=Z^{(4,1)}=I_{\infty}, & Z^{(1,1)}=Z^{(2,2)}=\operatorname{superdiag}\left((1,1, \ldots)^{\top}\right), \\
Z^{(2,1)}=\operatorname{diag}\left((0,1, \ldots)^{\top}\right), & Z^{(3,1)}=Z^{(4,2)}=\operatorname{subdiag}\left((1,2, \ldots)^{\top}\right) .
\end{array}
$$

Next, the state space partitions from Definition 5 are computed as

$$
s_{1}^{(l, 1)}=\{l\}, \quad s_{2}^{(l, 1)}=\{0, \ldots, l\}, \quad s_{1}^{(l, 2)}=\{0, \ldots, l-1\}, \quad s_{2}^{(l, 2)}=\{l\},
$$

and, therefore,

$$
\begin{aligned}
& s^{(l, 1)}=s_{1}^{(l, 1)} \times s_{2}^{(l, 1)}=\{(l, 0), \ldots,(l, l)\}, \\
& s^{(l, 2)}=s_{1}^{(l, 2)} \times s_{2}^{(l, 2)}=\{(0, l), \ldots,(l-1, l)\} .
\end{aligned}
$$

Finally, since $n_{\mathrm{I}}=2$, from Table 1 and Definition 6, the nonzero blocks $Q_{0,0}, Q_{0,1}, Q_{1,0}$, and $Q_{l, m}$ for $l>0$ and $m \in\{l-1, l, l+1\}$ are respectively $(1 \times 1),(1 \times 2),(2 \times 1)$, and $(2 \times 2)$ block matrices.

Example 2. (Metabolite synthesis with two metabolites and one enzyme.) Consider a system of stochastic chemical kinetics modeling the biological process of metabolite synthesis with two metabolites and one enzyme [28]. The transition classes of this system are given in Table 2 . Here, $n=n_{\mathrm{I}}=3, n_{\mathrm{F}}=0, x=\left(x_{1}, x_{2}, x_{3}\right), J=7$, and $k_{A}, k_{B}, K_{I}, k_{2}, \mu, K_{R}, k_{E_{A}} \in \mathbb{R}_{>0}$. Hence, we have $\varsigma_{1}=\varsigma_{2}=\varsigma_{3}=\mathbb{Z}_{+},|\bar{\wp}|=1$, and $\delta=\varsigma_{1} \times \wp_{2} \times \wp_{3}$. Note that (2) implies $3 l^{2}+3 l+1$ states at level $l \in \mathbb{Z}_{+}$.

TABLE 1: Transition classes of the gene expression model.

\begin{tabular}{ccccc}
\hline$j$ & $\psi^{(j)}$ & $h^{(j, 1)}\left(x_{1}\right)$ & $h^{(j, 2)}\left(x_{2}\right)$ & $v^{(j)}$ \\
\hline 1 & $\lambda$ & 1 & 1 & $e_{1}^{\top}$ \\
2 & $\mu$ & $x_{1}$ & 1 & $e_{2}^{\top}$ \\
3 & $\delta_{1}$ & $x_{1}$ & 1 & $-e_{1}^{\top}$ \\
4 & $\delta_{2}$ & 1 & $x_{2}$ & $-e_{2}^{\top}$ \\
\hline
\end{tabular}


TABLE 2: Transition classes of the molecule synthesis model with one enzyme.

\begin{tabular}{cccccc}
\hline$j$ & $\psi^{(j)}$ & $h^{(j, 1)}\left(x_{1}\right)$ & $h^{(j, 2)}\left(x_{2}\right)$ & $h^{(j, 3)}\left(x_{3}\right)$ & $v^{(j)}$ \\
\hline 1 & $k_{A} K_{I}$ & $\frac{1}{x_{1}+K_{I}}$ & 1 & $x_{3}$ & $e_{1}^{\top}$ \\
2 & $k_{B}$ & 1 & 1 & 1 & $e_{2}^{\top}$ \\
3 & $k_{2}$ & $x_{1}$ & $x_{2}$ & 1 & $\left(-e_{1}-e_{2}\right)^{\top}$ \\
4 & $\mu$ & $x_{1}$ & 1 & 1 & $-e_{1}^{\top}$ \\
5 & $\mu$ & 1 & $x_{2}$ & 1 & $-e_{2}^{\top}$ \\
6 & $k_{E_{A}} K_{R}$ & 1 & 1 & 1 & $e_{3}^{\top}$ \\
7 & $\mu$ & $x_{1}+K_{R}$ & 1 & $x_{3}$ & $-e_{3}^{\top}$ \\
\hline
\end{tabular}

The transition rate matrices of the model from Table 2 and Definition 2 are obtained as

$$
\begin{aligned}
Z^{(1,2)} & =Z^{(2,1)}=Z^{(2,3)}=Z^{(3,3)}=Z^{(4,2)}=Z^{(4,3)}=Z^{(5,1)}=Z^{(5,3)}=Z^{(6,2)} \\
& =Z^{(7,1)}=Z^{(7,2)}=I_{\infty}, \\
Z^{(1,1)} & =\operatorname{superdiag}\left(\left(\frac{1}{K_{I}}, \frac{1}{1+K_{I}}, \ldots\right)^{\top}\right), \\
Z^{(1,3)} & =\operatorname{diag}\left((0,1, \ldots)^{\top}\right), \\
Z^{(2,2)} & =Z^{(6,3)}=\operatorname{superdiag}\left((1,1, \ldots)^{\top}\right), \\
Z^{(6,1)} & =\operatorname{diag}\left(\left(\frac{1}{K_{R}}, \frac{1}{1+K_{R}}, \ldots\right)^{\top}\right), \\
Z^{(3,1)} & =Z^{(3,2)}=Z^{(4,1)}=Z^{(5,2)}=Z^{(7,3)}=\operatorname{subdiag}\left((1,2, \ldots)^{\top}\right) .
\end{aligned}
$$

The state space partitions from Definition 5 are computed as

$$
\begin{gathered}
s_{1}^{(l, 1)}=\{l\}, \quad s_{2}^{(l, 1)}=s_{3}^{(l, 1)}=\{0, \ldots, l\}, \\
s_{1}^{(l, 2)}=\{0, \ldots, l-1\}, \quad s_{2}^{(l, 2)}=\{l\}, \quad s_{3}^{(l, 2)}=\{0, \ldots, l\}, \\
s_{1}^{(l, 3)}=s_{2}^{(l, 3)}=\{0, \ldots, l-1\}, \quad s_{3}^{(l, 3)}=\{l\},
\end{gathered}
$$

and, therefore,

$$
\begin{aligned}
& f^{(l, 1)}=\underset{i=1}{\times} s_{i}^{(l, 1)}=\{(l, 0,0), \ldots,(l, l, l)\}, \\
& 8^{(l, 2)}=\underset{i=1}{\stackrel{3}{\times}} s_{i}^{(l, 2)}=\{(0, l, 0), \ldots,(l-1, l, l)\}, \\
& 8^{(l, 3)}=\underset{i=1}{\times} s_{i}^{(l, 3)}=\{(0,0, l), \ldots,(l-1, l-1, l)\} .
\end{aligned}
$$

Finally, since $n_{\mathrm{I}}=3$, from Table 2 and Definition 6, the nonzero blocks $Q_{0,0}, Q_{0,1}, Q_{1,0}$, and $Q_{l, m}$ for $l>0$ and $m \in\{l-1, l, l+1\}$ are respectively $(1 \times 1),(1 \times 3),(3 \times 1)$, and $(3 \times 3)$ block matrices. The first few nonzero blocks of $Q$ as flat sparse matrices and blocks of $Q_{l, l-1}, Q_{l, l}$, and $Q_{l, l+1}$ can be found in Appendix A. 
TABLE 3: Transition classes of the molecule synthesis model with two enzymes.

\begin{tabular}{|c|c|c|c|c|c|c|}
\hline$j$ & $\psi^{(j)}$ & $h^{(j, 1)}\left(x_{1}\right)$ & $h^{(j, 2)}\left(x_{2}\right)$ & $h^{(j, 3)}\left(x_{3}\right)$ & $h^{(j, 4)}\left(x_{4}\right)$ & $v^{(j)}$ \\
\hline 1 & $k_{A} K_{I}$ & $\frac{1}{x_{1}+K_{I}}$ & 1 & $x_{3}$ & 1 & $e_{1}^{\top}$ \\
\hline 2 & $k_{B} K_{I}$ & 1 & $\frac{1}{x_{2}+K_{I}}$ & 1 & $x_{4}$ & $e_{2}^{\top}$ \\
\hline 3 & $k_{2}$ & $x_{1}$ & $x_{2}$ & 1 & 1 & $\left(-e_{1}-e_{2}\right)^{\top}$ \\
\hline 4 & $\mu$ & $x_{1}$ & 1 & 1 & 1 & $-e_{1}^{\top}$ \\
\hline 5 & $\mu$ & 1 & $x_{2}$ & 1 & 1 & $-e_{2}^{\top}$ \\
\hline 6 & $k_{E_{A}} K_{R}$ & $\frac{1}{x_{1}+K_{R}}$ & 1 & 1 & 1 & $e_{3}^{\top}$ \\
\hline 7 & $k_{E_{B}} K_{R}$ & 1 & $\frac{1}{x_{2}+K_{R}}$ & 1 & 1 & $e_{4}^{\top}$ \\
\hline 8 & $\mu$ & 1 & 1 & $x_{3}$ & 1 & $-e_{3}^{\top}$ \\
\hline 9 & $\mu$ & 1 & 1 & 1 & $x_{4}$ & $-e_{4}^{\top}$ \\
\hline
\end{tabular}

Example 3. (Metabolite synthesis with two metabolites and two enzymes.) Consider a system of stochastic chemical kinetics modeling the biological process of metabolite synthesis with two metabolites and two enzymes [28]. The transition classes of this system are given in Table 3 . Here, $n=n_{\mathrm{I}}=4, n_{\mathrm{F}}=0, x=\left(x_{1}, x_{2}, x_{3}, x_{4}\right), J=9$, and $k_{A}, k_{B}, K_{I}, k_{2}, \mu, K_{R}, k_{E_{A}}$,

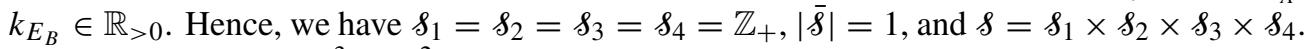
Note that (2) implies $4 l^{3}+6 l^{2}+4 l+1$ states at level $l \in \mathbb{Z}_{+}$.

The transition rate matrices of the model from Table 3 and Definition 2 are obtained as

$$
\begin{aligned}
Z^{(1,2)} & =Z^{(1,4)}=Z^{(2,1)}=Z^{(2,3)}=Z^{(3,3)}=Z^{(3,4)}=Z^{(4,2)}=Z^{(4,3)}=Z^{(4,4)} \\
& =Z^{(5,1)}=Z^{(5,3)}=Z^{(5,4)}=Z^{(6,2)}=Z^{(6,4)}=Z^{(7,1)}=Z^{(7,3)}=Z^{(8,1)} \\
& =Z^{(8,2)}=Z^{(8,4)}=Z^{(9,1)}=Z^{(9,2)}=Z^{(9,3)}=I_{\infty}, \\
Z^{(1,1)} & =Z^{(2,2)}=\operatorname{superdiag}\left(\left(\frac{1}{K_{I}}, \frac{1}{1+K_{I}}, \ldots\right)^{\top}\right), \\
Z^{(1,3)} & =Z^{(2,4)}=\operatorname{diag}\left((0,1, \ldots)^{\top}\right), \\
Z^{(6,3)} & =Z^{(7,4)}=\operatorname{superdiag}\left((1,1, \ldots)^{\top}\right), \\
Z^{(6,1)} & =Z^{(7,2)}=\operatorname{diag}\left(\left(\frac{1}{K_{R}}, \frac{1}{1+K_{R}}, \ldots\right)^{\top}\right), \\
Z^{(3,1)} & =Z^{(3,2)}=Z^{(4,1)}=Z^{(5,2)}=Z^{(8,3)}=Z^{(9,4)}=\operatorname{subdiag}\left((1,2, \ldots)^{\top}\right) .
\end{aligned}
$$

The state space partitions from Definition 5 are computed as

$$
\begin{gathered}
s_{1}^{(l, 1)}=\{l\}, \quad s_{2}^{(l, 1)}=s_{3}^{(l, 1)}=s_{4}^{(l, 1)}=\{0, \ldots, l\}, \\
s_{1}^{(l, 2)}=\{0, \ldots, l-1\}, \quad s_{2}^{(l, 2)}=\{l\}, \quad s_{3}^{(l, 2)}=s_{4}^{(l, 2)}=\{0, \ldots, l\}, \\
s_{1}^{(l, 3)}=s_{2}^{(l, 3)}=\{0, \ldots, l-1\}, \quad s_{3}^{(l, 3)}=\{l\}, \quad s_{4}^{(l, 3)}=\{0, \ldots, l\}, \\
s_{1}^{(l, 4)}=s_{2}^{(l, 4)}=s_{3}^{(l, 4)}=\{0, \ldots, l-1\}, \quad s_{4}^{(l, 4)}=\{l\},
\end{gathered}
$$


TABLE 4: Transition classes of the molecule synthesis model with repressilator.

\begin{tabular}{ccccccccc}
\hline$j$ & $\psi^{(j)}$ & $h^{(j, 1)}\left(x_{1}\right) h^{(j, 2)}\left(x_{2}\right)$ & $h^{(j, 3)}\left(x_{3}\right)$ & $h^{(j, 4)}\left(x_{4}\right)$ & $h^{(j, 5)}\left(x_{5}\right)$ & $h^{(j, 6)}\left(x_{6}\right)$ & $v^{(j)}$ \\
\hline 1 & $\lambda_{1}$ & 1 & 1 & 1 & 1 & 1 & $1-x_{6}$ & $e_{1}^{\top}$ \\
2 & $\delta_{1}$ & $x_{1}$ & 1 & 1 & 1 & 1 & 1 & $-e_{1}^{\top}$ \\
3 & $\beta_{0}$ & $x_{1}$ & 1 & 1 & $1-x_{4}$ & 1 & 1 & $\left(-e_{1}+e_{4}\right)^{\top}$ \\
4 & $\beta_{1}$ & 1 & 1 & 1 & $x_{4}$ & 1 & 1 & $\left(e_{1}-e_{4}\right)^{\top}$ \\
5 & $\lambda_{2}$ & 1 & 1 & 1 & $1-x_{4}$ & 1 & 1 & $e_{2}^{\top}$ \\
6 & $\delta_{2}$ & 1 & $x_{2}$ & 1 & 1 & 1 & 1 & $-e_{2}^{\top}$ \\
7 & $\beta_{0}$ & 1 & $x_{2}$ & 1 & 1 & $1-x_{5}$ & 1 & $\left(-e_{2}+e_{5}\right)^{\top}$ \\
8 & $\beta_{1}$ & 1 & 1 & 1 & 1 & $x_{5}$ & 1 & $\left(e_{2}-e_{5}\right)^{\top}$ \\
9 & $\lambda_{3}$ & 1 & 1 & 1 & 1 & $1-x_{5}$ & 1 & $e_{3}^{\top}$ \\
10 & $\delta_{3}$ & 1 & 1 & $x_{3}$ & 1 & 1 & 1 & $-e_{3}^{\top}$ \\
11 & $\beta_{0}$ & 1 & 1 & $x_{3}$ & 1 & 1 & $1-x_{6}$ & $\left(-e_{3}+e_{6}\right)^{\top}$ \\
12 & $\beta_{1}$ & 1 & 1 & 1 & 1 & 1 & $x_{6}$ & $\left(e_{3}-e_{6}\right)^{\top}$ \\
\hline
\end{tabular}

and, therefore,

$$
\begin{aligned}
& s^{(l, 1)}=\underset{i=1}{\times} s_{i}^{(l, 1)}=\{(l, 0,0,0), \ldots,(l, l, l, l)\} \\
& s^{(l, 2)}=\underset{i=1}{\stackrel{4}{\times}} s_{i}^{(l, 2)}=\{(0, l, 0,0), \ldots,(l-1, l, l, l)\}, \\
& s^{(l, 3)}=\underset{i=1}{\stackrel{4}{\times}} s_{i}^{(l, 3)}=\{(0,0, l, 0), \ldots,(l-1, l-1, l, l)\}, \\
& s^{(l, 4)}=\underset{i=1}{4} s_{i}^{(l, 4)}=\{(0,0,0, l), \ldots,(l-1, l-1, l-1, l)\} .
\end{aligned}
$$

Finally, since $n_{\mathrm{I}}=4$, from Table 3 and Definition 6, the nonzero blocks $Q_{0,0}, Q_{0,1}, Q_{1,0}$, and $Q_{l, m}$ for $l>0$ and $m \in\{l-1, l, l+1\}$ are respectively $(1 \times 1),(1 \times 4),(4 \times 1)$, and $(4 \times 4)$ block matrices.

The following example is different to the first three in that it has a control unit of eight states.

Example 4. (Repressilator.) Consider a system of stochastic chemical kinetics modeling the biological process of metabolite synthesis with repressilator [18]. The transition classes of this system are given in Table 4. Here, $n=6, n_{\mathrm{I}}=3, n_{\mathrm{F}}=3, x=\left(x_{1}, x_{2}, x_{3}, x_{4}, x_{5}, x_{6}\right)$, $J=12$, and $\lambda_{1}, \lambda_{2}, \lambda_{3}, \delta_{1}, \delta_{2}, \delta_{3}, \beta_{0}, \beta_{1} \in \mathbb{R}_{>0}$. Hence, we have $\varsigma_{1}=\varsigma_{2}=\varsigma_{3}=\mathbb{Z}_{+}$,

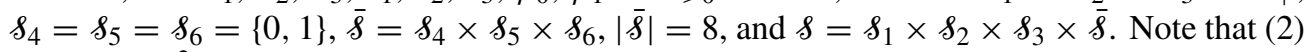
implies $8\left(3 l^{2}+3 l+1\right)$ states at level $l \in \mathbb{Z}_{+}$.

The transition rate matrices of the model from Table 4 and Definition 2 are obtained as

$$
\begin{aligned}
Z^{(1,2)} & =Z^{(1,3)}=Z^{(2,2)}=Z^{(2,3)}=Z^{(3,2)}=Z^{(3,3)}=Z^{(4,2)}=Z^{(4,3)}=Z^{(5,1)}=Z^{(5,3)} \\
& =Z^{(6,1)}=Z^{(6,3)}=Z^{(7,1)}=Z^{(7,3)}=Z^{(8,1)}=Z^{(8,3)}=Z^{(9,1)}=Z^{(9,2)}=Z^{(10,1)} \\
& =Z^{(10,2)}=Z^{(11,1)}=Z^{(11,2)}=Z^{(12,1)}=Z^{(12,2)}=I_{\infty}, \\
Z^{(1,1)} & =Z^{(4,1)}=Z^{(5,2)}=Z^{(8,2)}=Z^{(9,3)}=Z^{(12,3)}=\operatorname{superdiag}\left((1,1, \ldots)^{\top}\right), \\
Z^{(2,1)} & =Z^{(3,1)}=Z^{(6,2)}=Z^{(7,2)}=Z^{(10,3)}=Z^{(11,3)}=\operatorname{subdiag}\left((1,2, \ldots)^{\top}\right) .
\end{aligned}
$$


The joint transition rate matrices of the model for finite variables from Table 4 and Definition 3 are obtained as

$$
\begin{array}{lll}
\bar{Z}^{(2)}=\bar{Z}^{(6)}=\bar{Z}^{(10)}=I_{2} \otimes I_{2} \otimes I_{2}, & & \bar{Z}^{(1)}=I_{2} \otimes I_{2} \otimes \operatorname{diag}\left((1,0)^{\top}\right), \\
\bar{Z}^{(3)}=\operatorname{superdiag}\left((1)^{\top}\right) \otimes I_{2} \otimes I_{2}, & & \bar{Z}^{(4)}=\operatorname{subdiag}\left((1)^{\top}\right) \otimes I_{2} \otimes I_{2}, \\
\bar{Z}^{(5)}=\operatorname{diag}\left((1,0)^{\top}\right) \otimes I_{2} \otimes I_{2}, & & \bar{Z}^{(7)}=I_{2} \otimes \operatorname{superdiag}\left((1)^{\top}\right) \otimes I_{2}, \\
\bar{Z}^{(8)}=I_{2} \otimes \operatorname{subdiag}\left((1)^{\top}\right) \otimes I_{2}, & & \bar{Z}^{(9)}=I_{2} \otimes \operatorname{diag}\left((1,0)^{\top}\right) \otimes I_{2}, \\
\bar{Z}^{(11)}=I_{2} \otimes I_{2} \otimes \operatorname{superdiag}\left((1)^{\top}\right), & & \bar{Z}^{(12)}=I_{2} \otimes I_{2} \otimes \operatorname{subdiag}\left((1)^{\top}\right) .
\end{array}
$$

The state space partitions from Definition 5 are computed as in Example 2, but now we also have $\bar{s}=\{(0,0,0), \ldots,(1,1,1)\}$, and, therefore,

$$
\begin{aligned}
& s^{(l, 1)}=\left(\underset{i=1}{\underset{x}{x}} s_{i}^{(l, 1)}\right) \times \bar{s}=\{(l, 0,0,0,0,0), \ldots,(l, l, l, 1,1,1)\}, \\
& s^{(l, 2)}=\left(\underset{i=1}{\stackrel{3}{\times}} s_{i}^{(l, 2)}\right) \times \bar{s}=\{(0, l, 0,0,0,0), \ldots,(l-1, l, l, 1,1,1)\}, \\
& s^{(l, 3)}=\left(\underset{i=1}{\times} s_{i}^{(l, 3)}\right) \times \bar{s}=\{(0,0, l, 0,0,0), \ldots,(l-1, l-1, l, 1,1,1)\} .
\end{aligned}
$$

Finally, since $n_{\mathrm{I}}=3$, from Table 4 and Definition 6, the nonzero blocks $Q_{0,0}, Q_{0,1}, Q_{1,0}$, and $Q_{l, m}$ for $l>0$ and $m \in\{l-1, l, l+1\}$ are respectively $(1 \times 1),(1 \times 3),(3 \times 1)$, and $(3 \times 3)$ block matrices.

In the next section, after briefly recalling how we compute the low and high level numbers of the LDQBD process between which a specified percentage of the steady-state probability mass lies, we describe how the simulation is carried out. Then we provide the results of experiments on the four examples of this section.

\section{Numerical results}

It has been shown by Tweedie [32] that the LDQBD process is ergodic if and only if there exists a function $g: \& \rightarrow \mathbb{R}_{\geq 0}$, called a Lyapunov function, and a finite set $\mathcal{C} \subset \delta$ satisfying the three conditions

(i) $d(x) \leq-\gamma$ for all $x \in \delta \backslash \mathcal{C}$ and some $\gamma>0$,

(ii) $d(x)<\infty$ for all $x \in \mathcal{C}$, and

(iii) $\{x \in \delta \mid g(x) \leq r\}$ is finite for all $r<\infty$,

where

$$
d(x)=\sum_{j=1}^{J} \alpha_{j}(x)\left(g\left(x+v^{(j)}\right)-g(x)\right) \in \mathbb{R}
$$

is called the drift in state $x \in \&$.

Assuming that $g$ satisfies condition (iii) and letting $c=\sup _{x \in \delta} d(x)<\infty$, an upper bound on $\sum_{x \in S \backslash C} \pi(x)$ can be a priori specified as $\varepsilon=c /(c+\gamma) \in(0,1)$, which translates to $\gamma=c / \varepsilon-c$ and $\mathcal{C}=\{x \in \delta \mid d(x)>-\gamma\}$. In addition, if $\mathcal{C}$ is finite then the three conditions above hold and $\sum_{x \in \mathcal{C}} \pi(x) \geq 1-\varepsilon$. 
In order to determine $c$, the domain of the search for extrema is restricted to $\mathbb{R}_{\geq 0}^{1 \times n_{\mathrm{I}}}$ for infinite components and $\bar{s}$ for finite components. All extrema are computed by equating the gradient of $d(x)$ to 0 . In order to determine all local extrema including those located on the boundaries of the domain, the same system is solved for every projection of $d(x)$ onto each subspace of $\mathbb{R}^{1 \times n_{\mathrm{I}}}$ by setting all combinations of variables $x_{i}$ for $i \in\left\{1, \ldots, n_{\mathrm{I}}\right\}$ to 0 . Since $c$ is the supremum of the drift function over the state space, $\$$, we compute the drift at the states in the neighborhood of all extrema and choose its maximum value. Throughout this process, the resulting nonlinear equation systems are solved using the HOM4PS2 - 2.0 package [16], an implementation of the polyhedral homotopy continuation method. For details, see [6].

We compute the pair of level numbers, (low, high), of the LDQBD process such that the states in levels low to high include all the states in $\mathcal{C}$. In other words, we set

$$
\text { low }=\min \left\{l \in \mathbb{Z}_{+} \mid 8^{(l)} \cap \mathcal{C} \neq \varnothing\right\} \quad \text { and } \quad h i g h=\max \left\{l \in \mathbb{Z}_{+} \mid 8^{(l)} \cap \mathcal{C} \neq \varnothing\right\},
$$

and the finite set $\bigcup_{10 w \leq l \leq h i g h} f^{(l)}$ contains at least $1-\varepsilon$ of the steady-state probability. The number of states lying in levels low to high are given by

$$
N(\text { low, high })=\sum_{l=\text { low }}^{\text {high }}|\bar{s}|\left((l+1)^{n_{\mathrm{I}}}-(l)^{n_{\mathrm{I}}}\right)=|\bar{s}|\left((\text { high }+1)^{n_{\mathrm{I}}}-(\text { low })^{n_{\mathrm{I}}}\right) .
$$

In order to carry out a fair comparison with simulation, for all examples, we choose the squared Euclidean norm, that is, $g(x)=\sum_{i=1}^{n} x_{i}^{2}$, as the Lyapunov function and set $\varepsilon=0.05$. In other words, the results we present with the LDQBD solver [5] developed in MATLAB ${ }^{\circledR}$ are not fine tuned. In [7], it has already been shown that, for smaller $\varepsilon$, the accuracy of the computed solution by the matrix-analytic approach improves and eventually reaches that of machine precision. We do not undertake such a study here due to memory limitations imposed by the multidimensional models we consider. Given more memory (and time), it is always possible to obtain more accurate results with the matrix-analytic approach. We will see what we get with $\varepsilon=0.05$.

The proof that $\mathcal{C}$ is finite (if possible) follows a line of argument which constructively defines a finite superset of $\mathcal{C}$. After considering the form of the drift functions in our examples due to the choice of the squared Euclidean norm as the Lyapunov function, we define $n_{\text {I quadratic }}$ polynomials

$$
f_{i}\left(x_{i}\right)=a_{2, i} x_{i}^{2}+a_{1, i} x_{i}+a_{0, i} \quad \text { with } \quad a_{2, i}<0 \quad \text { for } i \in\left\{1, \ldots, n_{\mathrm{I}}\right\}
$$

so as to satisfy

$$
d(x) \leq \sum_{i=1}^{n_{\mathrm{I}}} f_{i}\left(x_{i}\right) \quad \text { for } x=\left(x_{1}, \ldots, x_{n}\right) \in \S .
$$

Since $f_{i}\left(x_{i}\right)$ is concave down for $i \in\left\{1, \ldots, n_{\mathrm{I}}\right\}$ and $x \in \&$ by construction, the upper bound on $d(x)$ over $\&$ is finite; hence, $c=\sup _{x \in \delta} d(x)$ is finite. Now, consider adding a constant $\gamma \in \mathbb{R}_{\geq 0}$ to both of sides of the inequality so that

$$
0<\gamma+d(x) \leq \gamma+\sum_{i=1}^{n_{\mathrm{I}}} f_{i}\left(x_{i}\right), \quad \text { or, equivalently, } \quad-\gamma<d(x) \leq \sum_{i=1}^{n_{\mathrm{I}}} f_{i}\left(x_{i}\right) .
$$

The inequality $-\gamma<d(x)$ holds for some $x \in \delta$ and characterizes the set $\mathcal{C}$. That is, $\mathcal{C}=\{x \in \delta \mid d(x)>-\gamma\}$. This set clearly is a subset of $\mathcal{D}=\left\{x \in \& \mid-\gamma<\sum_{i=1}^{n_{\mathrm{I}}} f_{i}\left(x_{i}\right)\right\}$ 
from the last inequality; hence, we have $\mathcal{C} \subseteq \mathscr{D}$. It remains to show that $\mathscr{D}$ is finite. But, that is straightforward, because $f_{i}\left(x_{i}\right)$ is concave down and $x_{i} \in \mathbb{Z}_{+}$for $i \in\left\{1, \ldots, n_{\mathrm{I}}\right\}$.

In the following, we use subscripts as in $d_{p}, c_{p}, \gamma_{p}, \mathcal{C}_{p}, f_{p, i}$ for $i \in\left\{1, \ldots, n_{\mathrm{I}}\right\}$, (low $_{p}$, high $)$ ), and $N_{p}$ for Example $p \in\{1,2,3,4\}$, and report the values of $c_{p}$ and $\gamma_{p}$ in two decimal digits of precision.

Example 5. (Example 1 continued: gene expression.) We let $\lambda=\mu=\delta_{2}=0.05$ and $\delta_{1}=0.015$ be the parameters. Then the drift is given by

$$
d_{1}\left(x_{1}, x_{2}\right)=-0.03 x_{1}^{2}-0.1 x_{2}^{2}+0.1 x_{1} x_{2}+0.165 x_{1}+0.05 x_{2}+0.05 .
$$

Finiteness of $c_{1}$ and $\mathcal{C}_{1}$ can be shown by selecting $f_{1,1}\left(x_{1}\right)$ and $f_{1,2}\left(x_{2}\right)$ as

$$
f_{1,1}\left(x_{1}\right)=-0.001 x_{1}^{2}+0.165 x_{1}+0.05, \quad f_{1,2}\left(x_{2}\right)=-0.005 x_{2}^{2}+0.05 x_{2} .
$$

Note that $d_{1}\left(x_{1}, x_{2}\right)-f_{1,1}\left(x_{1}\right)-f_{1,2}\left(x_{2}\right) \leq 0$ can be obtained by rotating the axes and eliminating the $x_{1} x_{2}$ term as discussed in [8] and [29]. By using the HOM4PS2 -2 . 0 package, we obtain the global maximum drift $c_{1}=1.86$. For $\varepsilon=0.05$, we compute $\gamma_{1}=35.25$, $\left(\right.$ low $_{1}$, high $\left._{1}\right)=(0,105)$, and $N_{1}(0,105)=11236$.

Example 6. (Example 2 continued: metabolite synthesis with two metabolites and one enzyme.) We let $k_{A}=k_{B}=0.3, K_{I}=16, k_{2}=0.05, \mu=0.1, K_{R}=8$, and $k_{E_{A}}=0.02$ be the parameters. Then the drift is given by

$$
\begin{aligned}
d_{2}\left(x_{1}, x_{2}, x_{3}\right)= & \frac{9.6 x_{1} x_{3}+4.8 x_{3}}{x_{1}+16}+\frac{0.32 x_{3}+0.16}{x_{1}+8}-0.1 x_{1}^{2} x_{2}-0.1 x_{1} x_{2}^{2}+0.1 x_{1} x_{2} \\
& -0.2 x_{1}^{2}-0.2 x_{2}^{2}-0.2 x_{3}^{2}+0.1 x_{1}+0.7 x_{2}+0.1 x_{3}+0.3 .
\end{aligned}
$$

Finiteness of $c_{2}$ and $\mathcal{C}_{2}$ can be shown by selecting $f_{2,1}\left(x_{1}\right), f_{2,2}\left(x_{2}\right)$, and $f_{2,3}\left(x_{3}\right)$ as

$$
\begin{aligned}
& f_{2,1}\left(x_{1}\right)=-0.2 x_{1}^{2}+0.1 x_{1}+0.3 \\
& f_{2,2}\left(x_{2}\right)=-0.2 x_{2}^{2}+0.7 x_{2}, \\
& f_{2,3}\left(x_{3}\right)=-0.2 x_{3}^{2}+14.82 x_{3}+0.16 .
\end{aligned}
$$

The HOM4PS2 - 2 . 0 package requires the equation systems to consist of polynomials. Therefore we put the partial derivatives of $x_{1}$ and $x_{3}$ over a common denominator. We use the numerator of the derivative as input since the denominator is always positive for the parameters chosen. Then we obtain the global maximum drift $c_{2}=4.63$. For $\varepsilon=0.05$, we compute $\gamma_{2}=87.89,\left(\right.$ low $_{2}$, high $\left._{2}\right)=(0,31)$, and $N_{2}(0,31)=32768$.

Example 7. (Example 3 continued: metabolite synthesis with two metabolites and two enzymes.) We let $k_{A}=k_{B}=0.3, K_{I}=16, k_{2}=0.05, \mu=0.2, K_{R}=8$, and $k_{E_{A}}=k_{E_{B}}=0.02$ be the parameters. Then the drift is given by

$$
\begin{aligned}
d_{3}\left(x_{1}, x_{2}, x_{3}, x_{4}\right)= & \frac{9.6 x_{1} x_{3}+4.8 x_{3}}{x_{1}+16}+\frac{0.32 x_{3}+0.16}{x_{1}+8}+\frac{9.6 x_{2} x_{4}+4.8 x_{4}}{x_{2}+16} \\
& +\frac{0.32 x_{4}+0.16}{x_{2}+8}-0.1 x_{1}^{2} x_{2}-0.1 x_{1} x_{2}^{2}+0.1 x_{1} x_{2}-0.4 x_{1}^{2} \\
& -0.4 x_{2}^{2}-0.4 x_{3}^{2}-0.4 x_{4}^{2}+0.2 x_{1}+0.2 x_{2}+0.2 x_{3}+0.2 x_{4} .
\end{aligned}
$$


Finiteness of $c_{3}$ and $\mathcal{C}_{3}$ can be shown by selecting $f_{3,1}\left(x_{1}\right), f_{3,2}\left(x_{2}\right), f_{3,3}\left(x_{3}\right)$, and $f_{3,4}\left(x_{4}\right)$ as

$$
\begin{array}{cl}
f_{3,1}\left(x_{1}\right)=-0.4 x_{1}^{2}+0.2 x_{1}, & f_{3,2}\left(x_{2}\right)=-0.4 x_{2}^{2}+0.2 x_{2}, \\
f_{3,3}\left(x_{3}\right)=-0.4 x_{3}^{2}+14.92 x_{3}+1.6, \quad f_{3,4}\left(x_{4}\right)=-0.4 x_{4}^{2}+14.92 x_{4}+1.6 .
\end{array}
$$

We proceed as in the previous example and compute the global maximum drift $c_{3}=0.90$. For $\varepsilon=0.05$, we obtain $\gamma_{3}=17.11,\left(\right.$ low $_{3}$, high 3$)=(0,9)$, and $N_{3}(0,9)=10000$.

Example 8. (Example 4 continued: repressilator.) We let $\lambda_{1}=\lambda_{2}=\lambda_{3}=1.3, \delta_{1}=\delta_{2}=$ $\delta_{3}=0.8, \beta_{0}=1$, and $\beta_{1}=0.5$ be the parameters. Then the drift becomes

$$
\begin{aligned}
d_{4}\left(x_{1}, x_{2}, x_{3}, x_{4}, x_{5}, x_{6}\right)= & -3.6 x_{1}^{2}+2 x_{1}^{2} x_{4}-x_{1} x_{4}-2.6 x_{1} x_{6}+5.4 x_{1}-3.6 x_{2}^{2} \\
& +2 x_{2}^{2} x_{5}-x_{2} x_{5}-2.6 x_{2} x_{4}+5.4 x_{2}-3.6 x_{3}^{2}+2 x_{3}^{2} x_{6} \\
& -x_{3} x_{6}-2.6 x_{3} x_{5}+5.4 x_{3}-1.3 x_{4}-1.3 x_{5}-1.3 x_{6}+3.9 .
\end{aligned}
$$

Finiteness of $c_{4}$ and $\mathcal{C}_{4}$ can be shown by selecting $f_{4,1}\left(x_{1}\right), f_{4,2}\left(x_{2}\right)$, and $f_{4,3}\left(x_{3}\right)$ as

$$
\begin{aligned}
& f_{4,1}\left(x_{1}\right)=-1.6 x_{1}^{2}+5.4 x_{1}+1.3 \\
& f_{4,2}\left(x_{2}\right)=-1.6 x_{2}^{2}+5.4 x_{2}+1.3 \\
& f_{4,3}\left(x_{3}\right)=-1.6 x_{3}^{2}+5.4 x_{3}+1.3
\end{aligned}
$$

since $x_{4}, x_{5}$, and $x_{6}$ each take values from $\{0,1\}$. Again, by using the HOM4PS2 -2.0 package we obtain the global maximum drift $c_{4}=9.30$. For $\varepsilon=0.05$, we compute $\gamma_{4}=176.7$, $\left(\right.$ low $_{4}$, high $\left._{4}\right)=(0,12)$, and $N_{4}(0,12)=17576$.

The matrix-analytic approach used in the solution process is the one called A3 in [7]. That is, we start by setting the matrix of conditional expected sojourn times at level high to 0 [1] and compute the matrices of conditional expected sojourn times at levels $l \in\{$ low, .., high -1$\}$ recursively. Experiments are performed on an Intel ${ }^{\circledR} \mathrm{Core}^{\mathrm{TM}} 2$ Duo $1.83 \mathrm{GHz}$ processor having 4 Gigabytes (GB) of main memory. Although it cannot be used to its full extent, the large main memory is necessary to store the relatively dense matrices of conditional expected sojourn times at levels $l \in\{$ low, ..., high -1$\}$ (see Figure 1 for their nonzero densities in the four examples and note how dense they become as the level number moves towards low) and the temporary factors allocated by MATLAB while solving linear systems with coefficient matrices involving them. The existence of two cores in the CPU is not exploited for parallel computing in the implementation. Hence, only one of the two cores is busy running the solver in the experiments.

In Table 5, the first two columns give the example name and the corresponding (low, high) pair for the squared Euclidean norm as the Lyapunov function when we set $\varepsilon=0.05$. Hence, 95 is a lower bound on the percentage of the steady-state probability mass that lies in levels $l \in\{$ low, ..., high\}. Column 'Time' lists the time in seconds to solve the example with the LDQBD solver [5]. Column 'Residual' gives the infinity norm of the residual vector. Column 'Time' includes the time to compute the value in column 'Residual'. Finally, the last column gives the memory requirement in megabytes (MB) for the solution process associated with the particular example, excluding that taken by MATLAB itself. In the first two examples, we obtain a residual in the order of machine precision. In the last two examples, the results are still good and we have residuals in the order of at least $10^{-7}$. 


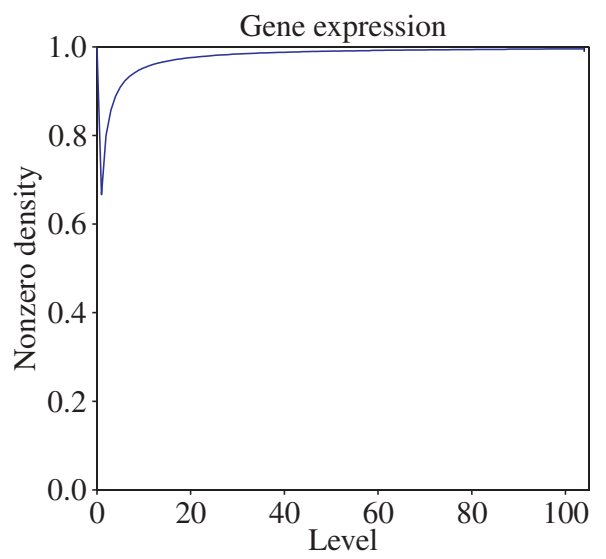

Metabolite synthesis with two metabolites

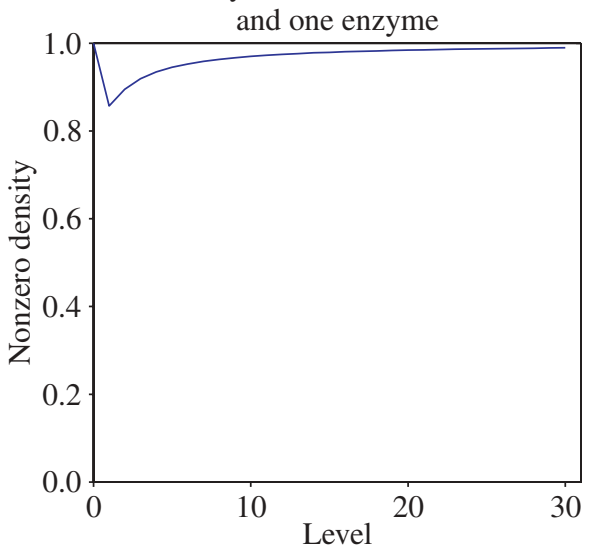

Metabolite synthesis with two metabolites
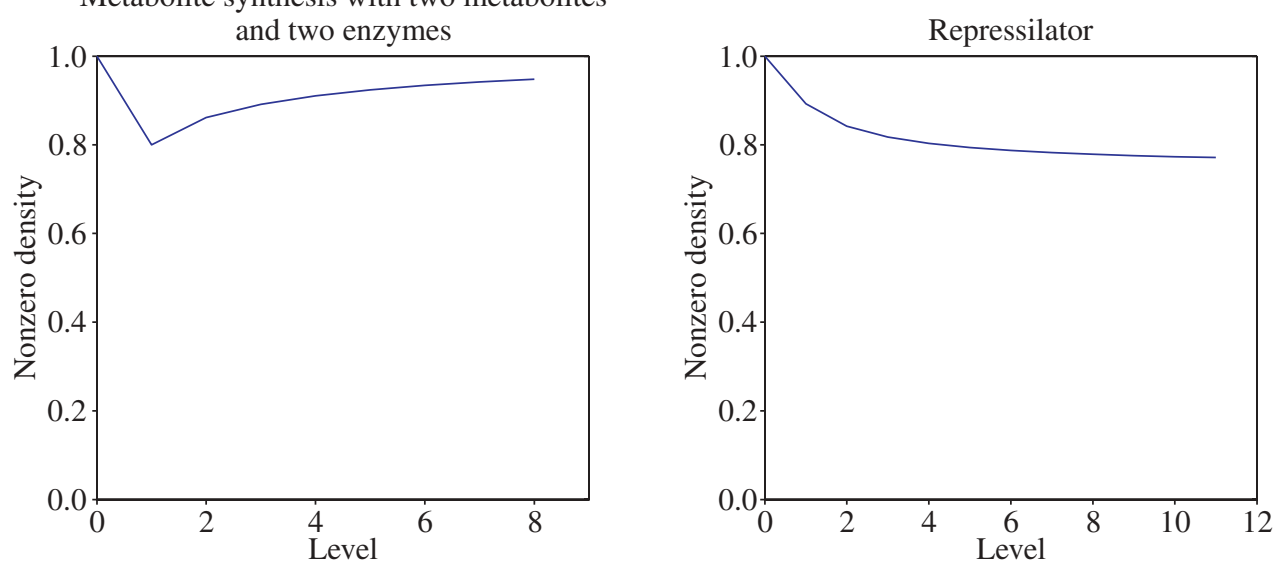

FIGURE 1: Nonzero densities in matrices of conditional expected sojourn times at levels $l \in\{$ low, ..., high $\}$ for the four examples.

TABLE 5: LDQBD solver results.

\begin{tabular}{lcccc}
\hline \multicolumn{1}{c}{ Example } & Tow, high) & $\begin{array}{c}\text { Time } \\
\text { (seconds) }\end{array}$ & Residual & $\begin{array}{c}\text { Memory } \\
\text { (MB) }\end{array}$ \\
\hline Gene expression & $(0,105)$ & 4 & $2 \times 10^{-17}$ & 20 \\
Molecule synthesis (one enzyme) & $(0,31)$ & 188 & $9 \times 10^{-17}$ & 667 \\
Molecule synthesis (two enzymes) & $(0,9)$ & 55 & $2 \times 10^{-7}$ & 180 \\
Repressilator & $(0,12)$ & 133 & $2 \times 10^{-8}$ & 335 \\
\hline
\end{tabular}

Now, let us turn to stochastic simulation [9] and its benchmark implementation StochKit [26] discussed in [17]. In order to provide confidence intervals, we take 31 sample paths for each example. Furthermore, it is always an issue when to terminate simulations. To that end, we terminate the simulation dynamically by comparing the absolute value of the update on the current mean with a user-specified tolerance as follows. Let the current value of the 
TABLE 6: StochKit simulation results.

\begin{tabular}{|c|c|c|c|c|c|c|c|}
\hline Example & $\begin{array}{c}\text { Reaction } \\
\text { count }\end{array}$ & $\begin{array}{c}\text { Time } \\
\text { (seconds) }\end{array}$ & Molecule & Mean $_{\text {SK }}$ & $\mathrm{CI}_{\mathrm{SK}}$ & Mean $_{\text {LDQBD }}$ & $\mathrm{RE}$ \\
\hline \multirow[t]{2}{*}{ Gene expression } & \multirow[t]{2}{*}{$2 \times 10^{9}$} & \multirow[t]{2}{*}{324} & $X_{1}$ & 3.33334 & 0.00095 & 3.33333 & $2 \times 10^{-5}$ \\
\hline & & & $X_{2}$ & 3.33345 & 0.00112 & 3.33333 & $4 \times 10^{-5}$ \\
\hline \multirow{3}{*}{$\begin{array}{l}\text { Molecule synthesis } \\
\text { (one enzyme) }\end{array}$} & \multirow[t]{3}{*}{$4 \times 10^{9}$} & \multirow[t]{3}{*}{784} & $X_{1}$ & 0.27258 & 0.00014 & 0.28097 & $3 \times 10^{-2}$ \\
\hline & & & $X_{2}$ & 2.74106 & 0.00032 & 2.73546 & $2 \times 10^{-3}$ \\
\hline & & & $X_{3}$ & 0.19459 & 0.00009 & 0.19442 & $9 \times 10^{-4}$ \\
\hline \multirow{4}{*}{$\begin{array}{l}\text { Molecule synthesis } \\
\text { (two enzymes) }\end{array}$} & \multirow[t]{4}{*}{$7 \times 10^{9}$} & \multirow[t]{4}{*}{1241} & $X_{1}$ & 0.13530 & 0.00003 & 0.13753 & $2 \times 10^{-2}$ \\
\hline & & & $X_{2}$ & 0.13529 & 0.00003 & 0.13753 & $2 \times 10^{-2}$ \\
\hline & & & $X_{3}$ & 0.09860 & 0.00001 & 0.09858 & $2 \times 10^{-4}$ \\
\hline & & & $X_{4}$ & 0.09860 & 0.00002 & 0.09858 & $2 \times 10^{-4}$ \\
\hline \multirow[t]{3}{*}{ Repressilator } & \multirow{3}{*}{$4 \times 10^{9}$} & \multirow[t]{3}{*}{761} & $X_{1}$ & 0.75708 & 0.00023 & 0.75701 & $1 \times 10^{-4}$ \\
\hline & & & $X_{2}$ & 0.75708 & 0.00019 & 0.75701 & $9 \times 10^{-5}$ \\
\hline & & & $X_{3}$ & 0.75699 & 0.00024 & 0.75701 & $2 \times 10^{-5}$ \\
\hline
\end{tabular}

StochKit (SK) mean for a particular molecule in a sample path be given by

$$
\operatorname{Mean}_{\mathrm{SK}}(K)=\sum_{k=1}^{K} S_{k} \Delta t_{k} / \sum_{k=1}^{K} \Delta t_{k}
$$

where $\Delta t_{k}$ is the exponentially distributed time of transition $k, S_{k}$ is the state of the molecule during $\Delta t_{k}$, and $K$ is the number of transitions taken up to this point. Then the new mean after transition $K+1$ takes place can be written as

$$
\operatorname{Mean}_{\mathrm{SK}}(K+1)=\operatorname{Mean}_{\mathrm{SK}}(K)+\left(S_{K+1}-\operatorname{Mean}_{\mathrm{SK}}(K)\right) \Delta t_{K+1} /\left(\Delta t_{K+1}+\sum_{k=1}^{K} \Delta t_{k}\right) .
$$

We remark that it is relatively easy to carry out the update on $\operatorname{Mean}_{S K}(K)$. And while it is computed, we can compare the absolute value of the update (that is, the second term) on the right-hand side of $\operatorname{Mean}_{\mathrm{SK}}(K+1)$ with the given tolerance. In our simulations, we have used $10^{-16}$ as the tolerance.

In Table 6, the first column gives the example name, the second column gives the total number of transitions that are taken for the entire course of the simulation (i.e. 31 sample paths), and column 'Time' lists the time in seconds for the simulation to terminate for the particular example. Column 'Molecule' indicates the identity of the molecule whose mean and confidence interval for a confidence probability of $95 \%$ are provided in the next two columns, named 'Mean SK' $_{\text {' and 'CI }}$ '. The mean computed by the LDQBD solution appears in the next to last column. Finally, in the last column the relative error (RE) in Mean $\mathrm{SK}_{\text {, }}$

$$
\mathrm{RE}=\frac{\left|\mathrm{Mean}_{\mathrm{LDQBD}}-\mathrm{Mean}_{\mathrm{SK}}\right|}{\text { Mean }_{\mathrm{LDQBD}}},
$$

is reported.

The memory requirement of the simulation is immaterial and therefore not reported. It is clear that the results are obtained with a higher accuracy in less time with the LDQBD solver for the problems considered here. 


\section{Conclusion}

We have provided a Kronecker representation for the nonzero blocks of the infinitesimal generator matrix underlying an infinite LDQBD process. The Kronecker representation seems to be necessary if the problem at hand is multidimensional. Thereafter, we have computed the mean number of molecules for examples from systems of stochastic chemical kinetics using the matrix-analytic LDQBD solver and stochastic simulation. The memory requirement of the LDQBD solver is incomparably large, but the accuracy of the results is much higher and the time to obtain the solution is much smaller compared to that of simulation. Future work should concentrate on extending the application domain of the LDQBD solver to queueing networks and trying to devise a Kronecker-based solver for the systems of linear equations at each level of the matrix-analytic approach. The latter should make the LDQBD solver even more scalable.

\section{Appendix A}

Using (1), Table 2, and Definitions 1 and 4, we obtain the first few nonzero blocks of $Q$ for Example 2 as flat sparse matrices given by

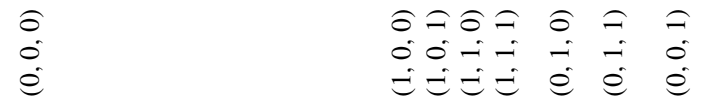

$$
\begin{aligned}
& Q_{0,0}=(0,0,0)(*), \quad Q_{0,1}=(0,0,0)\left(\quad\left|k_{B}\right| k_{E_{A}}\right),
\end{aligned}
$$

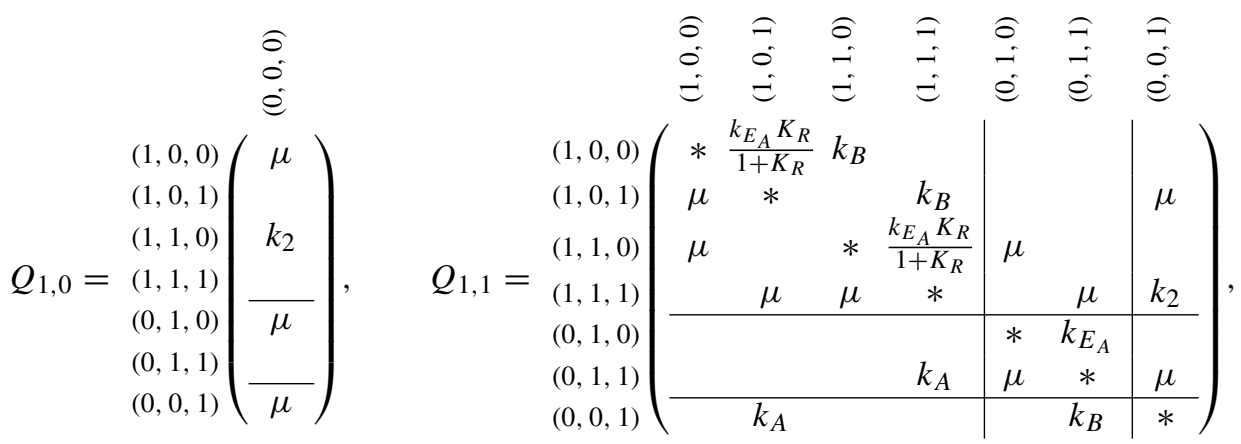

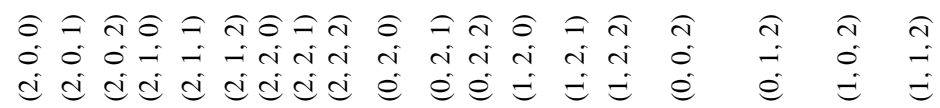

$$
\begin{aligned}
& \begin{array}{rl|l|l|l} 
& (1,0,0) \\
& (1,0,1) \\
& (1,1,0) \\
Q_{1,2}= & (1,1,1) \\
& (0,1,0) \\
& (0,1,1) \\
& (0,0,1)
\end{array}
\end{aligned}
$$




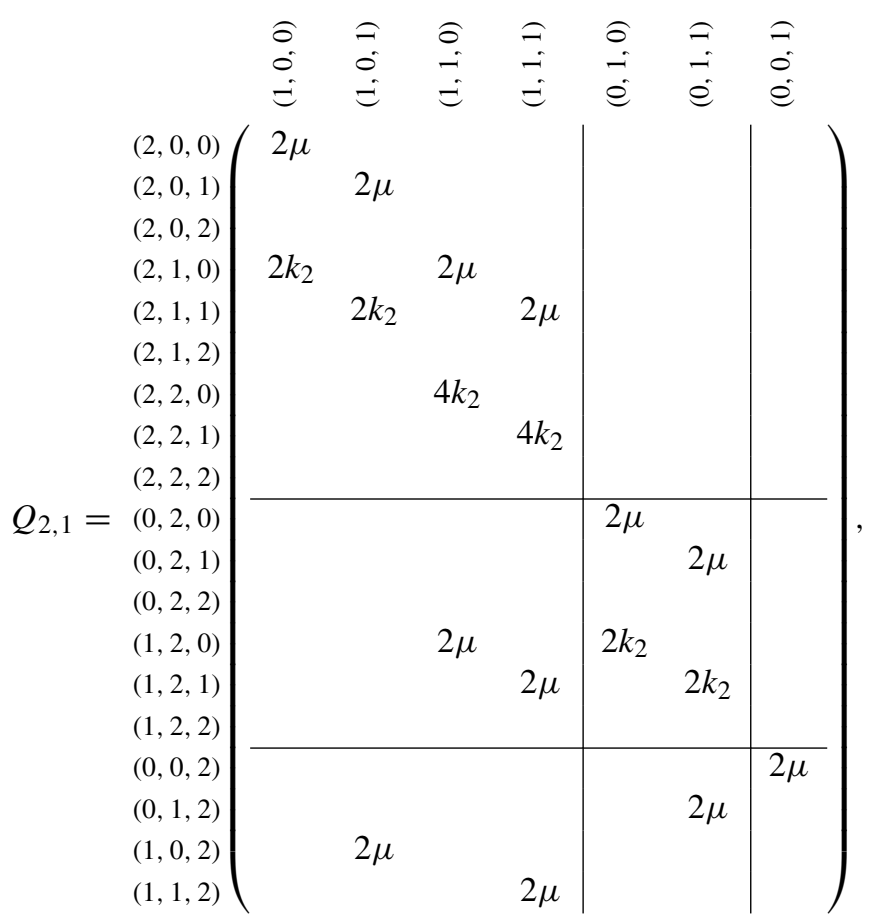

and $Q_{2,2}$ is given by

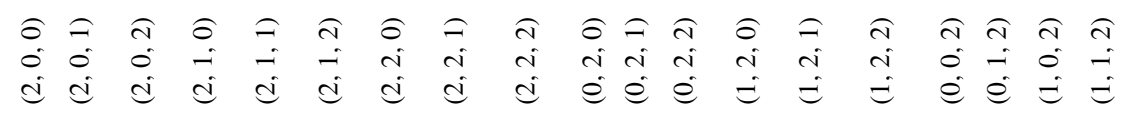

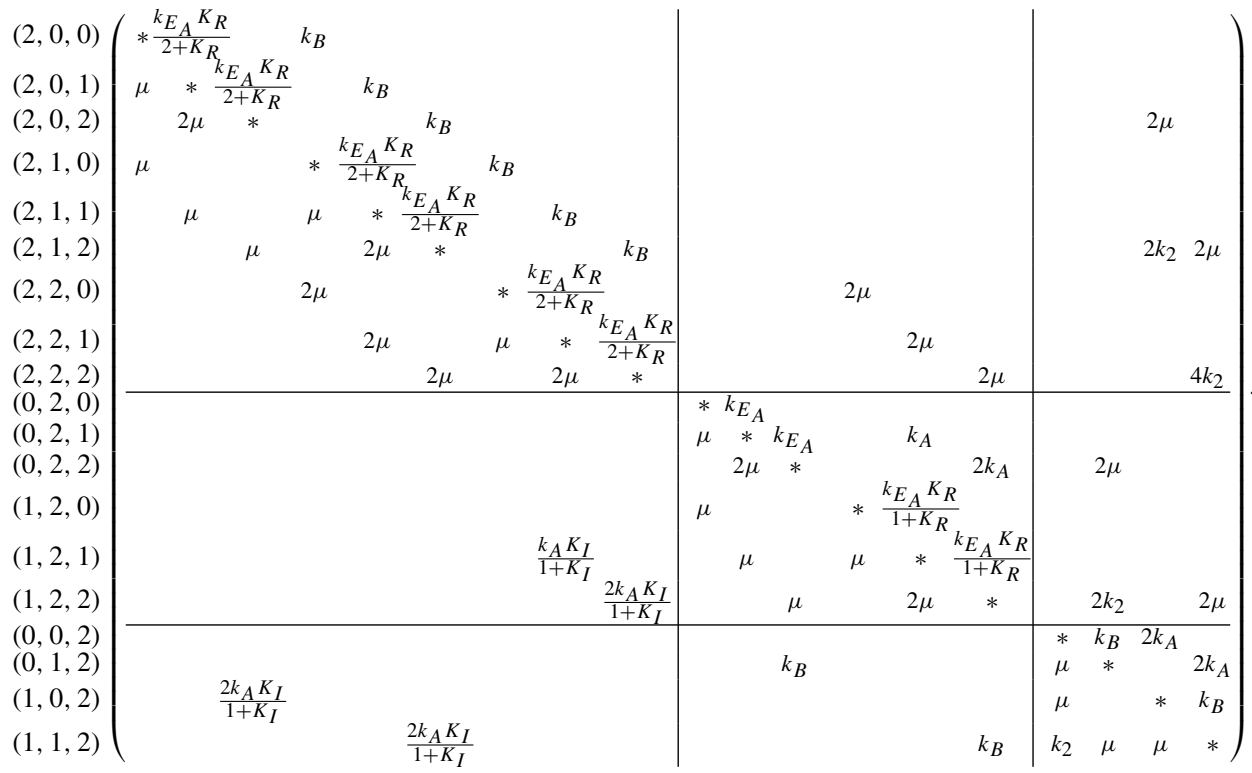


In particular, the nine blocks associated with $Q_{l, l-1}$ are obtained as $\tilde{Q}_{l, l-1}^{(1,1)}=\sum_{j=1}^{7} \psi^{(j)} Z^{(j, 1)}\left(f_{1}^{(l, 1)}, f_{1}^{(l-1,1)}\right) \otimes Z^{(j, 2)}\left(f_{2}^{(l, 1)}, f_{2}^{(l-1,1)}\right) \otimes Z^{(j, 3)}\left(f_{3}^{(l, 1)}, s_{3}^{(l-1,1)}\right)$ $=k_{2}(l) \otimes \operatorname{subdiag}\left((1, \ldots, l)^{\top}\right)_{(l+1) \times l} \otimes \operatorname{diag}\left((1, \ldots, 1)^{\top}\right)_{(l+1) \times l}$ $+\mu(l) \otimes \operatorname{diag}\left((1, \ldots, 1)^{\top}\right)_{(l+1) \times l} \otimes \operatorname{diag}\left((1, \ldots, 1)^{\top}\right)_{(l+1) \times l}$, $\tilde{Q}_{l, l-1}^{(1,2)}=\sum_{j=1}^{7} \psi^{(j)} Z^{(j, 1)}\left(\S_{1}^{(l, 1)}, \S_{1}^{(l-1,2)}\right) \otimes Z^{(j, 2)}\left(\wp_{2}^{(l, 1)}, \S_{2}^{(l-1,2)}\right) \otimes Z^{(j, 3)}\left(\S_{3}^{(l, 1)}, \S_{3}^{(l-1,2)}\right)$ $=0_{(l+1)^{2} \times(l-1) l}$,

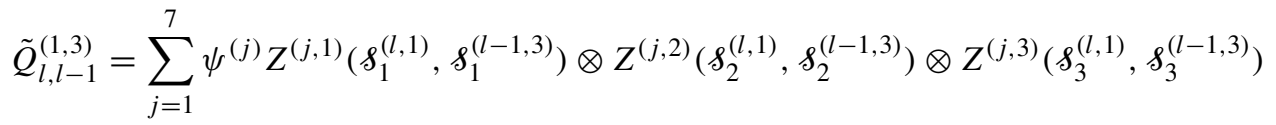
$=0_{(l+1)^{2} \times(l-1)^{2},}$

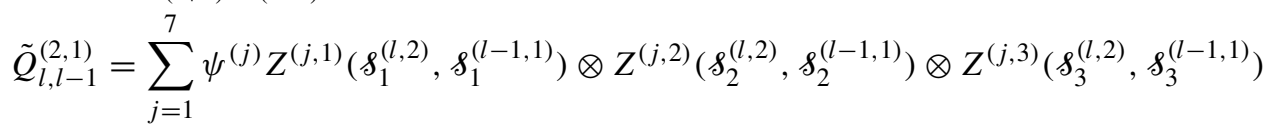
$=\mu(0, \ldots, 0,1)_{l \times 1}^{\top} \otimes(0, \ldots, 0, l)_{1 \times l} \otimes \operatorname{diag}\left((1, \ldots, 1)^{\top}\right)_{(l+1) \times l}$, $\tilde{Q}_{l, l-1}^{(2,2)}=\sum_{j=1}^{7} \psi^{(j)} Z^{(j, 1)}\left(f_{1}^{(l, 2)}, f_{1}^{(l-1,2)}\right) \otimes Z^{(j, 2)}\left(f_{2}^{(l, 2)}, s_{2}^{(l-1,2)}\right) \otimes Z^{(j, 3)}\left(f_{3}^{(l, 2)}, 8_{3}^{(l-1,2)}\right)$ $=k_{2} \operatorname{subdiag}\left((1, \ldots, l-1)^{\top}\right)_{l \times(l-1)} \otimes(l) \otimes \operatorname{diag}\left((1, \ldots, 1)^{\top}\right)_{(l+1) \times l}$ $+\mu \operatorname{diag}\left((1, \ldots, 1)^{\top}\right)_{l \times(l-1)} \otimes(l) \otimes \operatorname{diag}\left((1, \ldots, 1)^{\top}\right)_{(l+1) \times l}$, $\tilde{Q}_{l, l-1}^{(2,3)}=\sum_{j=1}^{7} \psi^{(j)} Z^{(j, 1)}\left(\S_{1}^{(l, 2)}, \S_{1}^{(l-1,3)}\right) \otimes Z^{(j, 2)}\left(\wp_{2}^{(l, 2)}, \S_{2}^{(l-1,3)}\right) \otimes Z^{(j, 3)}\left(f_{3}^{(l, 2)}, \wp_{3}^{(l-1,3)}\right)$ $=0_{l(l+1) \times(l-1)^{2}}$, $\tilde{Q}_{l, l-1}^{(3,1)}=\sum_{j=1}^{7} \psi^{(j)} Z^{(j, 1)}\left(f_{1}^{(l, 3)}, s_{1}^{(l-1,1)}\right) \otimes Z^{(j, 2)}\left(s_{2}^{(l, 3)}, s_{2}^{(l-1,1)}\right) \otimes Z^{(j, 3)}\left(f_{3}^{(l, 3)}, s_{3}^{(l-1,1)}\right)$ $=\mu(0, \ldots, 0,1)_{l \times 1}^{\top} \otimes \operatorname{diag}\left((1, \ldots, 1)^{\top}\right)_{l \times l} \otimes(0, \ldots, 0, l)_{1 \times l}$, $\tilde{Q}_{l, l-1}^{(3,2)}=\sum_{j=1}^{7} \psi^{(j)} Z^{(j, 1)}\left(s_{1}^{(l, 3)}, s_{1}^{(l-1,2)}\right) \otimes Z^{(j, 2)}\left(s_{2}^{(l, 3)}, s_{2}^{(l-1,2)}\right) \otimes Z^{(j, 3)}\left(s_{3}^{(l, 3)}, s_{3}^{(l-1,2)}\right)$ $=\mu \operatorname{diag}\left((1, \ldots, 1)^{\top}\right)_{l \times(l-1)} \otimes(0, \ldots, 0,1)_{l \times 1}^{\top} \otimes(0, \ldots, 0, l)_{1 \times l}$, $\tilde{Q}_{l, l-1}^{(3,3)}=\sum_{j=1}^{7} \psi^{(j)} Z^{(j, 1)}\left(\S_{1}^{(l, 3)}, \S_{1}^{(l-1,3)}\right) \otimes Z^{(j, 2)}\left(\S_{2}^{(l, 3)}, \S_{2}^{(l-1,3)}\right) \otimes Z^{(j, 3)}\left(f_{3}^{(l, 3)}, \S_{3}^{(l-1,3)}\right)$ $=\mu \operatorname{diag}\left((1, \ldots, 1)^{\top}\right)_{l \times(l-1)} \otimes \operatorname{diag}\left((1, \ldots, 1)^{\top}\right)_{l \times(l-1)} \otimes(l) ;$ the nine blocks associated with $Q_{l, l}$ are obtained as

$$
\begin{aligned}
\tilde{Q}_{l, l}^{(1,1)} & =\sum_{j=1}^{7} \psi^{(j)} Z^{(j, 1)}\left(\wp_{1}^{(l, 1)}, \varsigma_{1}^{(l, 1)}\right) \otimes Z^{(j, 2)}\left(f_{2}^{(l, 1)}, \wp_{2}^{(l, 1)}\right) \otimes Z^{(j, 3)}\left(f_{3}^{(l, 1)}, \wp_{3}^{(l, 1)}\right) \\
& =k_{B}(1) \otimes \operatorname{superdiag}\left((1, \ldots, 1)^{\top}\right)_{(l+1) \times(l+1)} \otimes \operatorname{diag}\left((1, \ldots, 1)^{\top}\right)_{(l+1) \times(l+1)}
\end{aligned}
$$


$+\mu(1) \otimes \operatorname{subdiag}\left((1, \ldots, l)^{\top}\right)_{(l+1) \times(l+1)} \otimes \operatorname{diag}\left((1, \ldots, 1)^{\top}\right)_{(l+1) \times(l+1)}$

$+k_{E_{A}} K_{R} \frac{1}{l+K_{R}}(1) \otimes \operatorname{diag}\left((1, \ldots, 1)^{\top}\right)_{(l+1) \times(l+1)}$

$\otimes \operatorname{superdiag}\left((1, \ldots, 1)^{\top}\right)_{(l+1) \times(l+1)}$

$+\mu(1) \otimes \operatorname{diag}\left((1, \ldots, 1)^{\top}\right)_{(l+1) \times(l+1)} \otimes \operatorname{subdiag}\left((1, \ldots, l)^{\top}\right)_{(l+1) \times(l+1)}$, $\tilde{Q}_{l, l}^{(1,2)}=\sum_{j=1}^{7} \psi^{(j)} Z^{(j, 1)}\left(\wp_{1}^{(l, 1)}, \wp_{1}^{(l, 2)}\right) \otimes Z^{(j, 2)}\left(\wp_{2}^{(l, 1)}, \wp_{2}^{(l, 2)}\right) \otimes Z^{(j, 3)}\left(f_{3}^{(l, 1)}, \S_{3}^{(l, 2)}\right)$ $=\mu(0, \ldots, 0, l)_{1 \times l} \otimes(0, \ldots, 0,1)_{(l+1) \times 1}^{\top} \otimes \operatorname{diag}\left((1, \ldots, 1)^{\top}\right)_{(l+1) \times(l+1)}$,

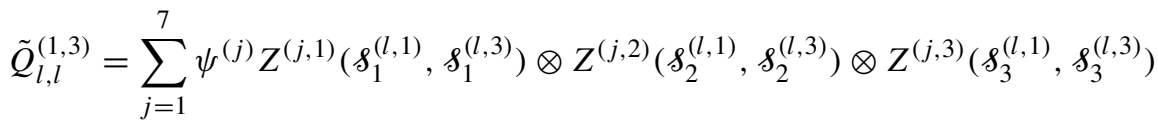
$=k_{2}(0, \ldots, 0, l)_{1 \times l} \otimes \operatorname{subdiag}\left((1, \ldots, l)^{\top}\right)_{(l+1) \times l} \otimes(0, \ldots, 0,1)_{(l+1) \times 1}^{\top}$ $+\mu(0, \ldots, 0, l)_{1 \times l} \otimes \operatorname{diag}\left((1, \ldots, 1)^{\top}\right)_{(l+1) \times l} \otimes(0, \ldots, 0,1)_{(l+1) \times 1}^{\top}$, $\tilde{Q}_{l, l}^{(2,1)}=\sum_{j=1}^{7} \psi^{(j)} Z^{(j, 1)}\left(\wp_{1}^{(l, 2)}, \wp_{1}^{(l, 1)}\right) \otimes Z^{(j, 2)}\left(f_{2}^{(l, 2)}, \wp_{2}^{(l, 1)}\right) \otimes Z^{(j, 3)}\left(f_{3}^{(l, 2)}, \jmath_{3}^{(l, 1)}\right)$ $=k_{A} K_{I}\left(0, \ldots, 0, \frac{1}{l-1+K_{I}}\right)_{l \times 1}^{\top} \otimes(0, \ldots, 0,1)_{1 \times(l+1)}$ $\otimes \operatorname{diag}\left((0, \ldots, l)^{\top}\right)_{(l+1) \times(l+1)}$, $\tilde{Q}_{l, l}^{(2,2)}=\sum_{j=1}^{7} \psi^{(j)} Z^{(j, 1)}\left(\S_{1}^{(l, 2)}, \wp_{1}^{(l, 2)}\right) \otimes Z^{(j, 2)}\left(\wp_{2}^{(l, 2)}, \S_{2}^{(l, 2)}\right) \otimes Z^{(j, 3)}\left(\S_{3}^{(l, 2)}, \S_{3}^{(l, 2)}\right)$ $=k_{A} K_{I} \operatorname{superdiag}\left(\left(\frac{1}{K_{I}}, \ldots, \frac{1}{l-2+K_{I}}\right)^{\top}\right)_{l \times l} \otimes(1)$ $\otimes \operatorname{diag}\left((0, \ldots, l)^{\top}\right)_{(l+1) \times(l+1)}$

$+\mu \operatorname{subdiag}\left((1, \ldots, l-1)^{\top}\right)_{l \times l} \otimes(1) \otimes \operatorname{diag}\left((1, \ldots, 1)^{\top}\right)_{(l+1) \times(l+1)}$ $+k_{E_{A}} K_{R} \operatorname{diag}\left(\left(\frac{1}{K_{R}}, \ldots, \frac{1}{l-1+K_{R}}\right)^{\top}\right)_{l \times l} \otimes(1)$ $\otimes \operatorname{superdiag}\left((1, \ldots, 1)^{\top}\right)_{(l+1) \times(l+1)}$

$+\mu \operatorname{diag}\left((1, \ldots, 1)^{\top}\right)_{l \times l} \otimes(1) \otimes \operatorname{subdiag}\left((1, \ldots, l)^{\top}\right)_{(l+1) \times(l+1)}$, $\tilde{Q}_{l, l}^{(2,3)}=\sum_{j=1}^{7} \psi^{(j)} Z^{(j, 1)}\left(8_{1}^{(l, 2)}, f_{1}^{(l, 3)}\right) \otimes Z^{(j, 2)}\left(f_{2}^{(l, 2)}, f_{2}^{(l, 3)}\right) \otimes Z^{(j, 3)}\left(f_{3}^{(l, 2)}, s_{3}^{(l, 3)}\right)$ $=k_{2} \operatorname{subdiag}\left((1, \ldots, l-1)^{\top}\right)_{l \times l} \otimes(0, \ldots, 0, l)_{1 \times l} \otimes(0, \ldots, 0,1)_{(l+1) \times 1}^{\top}$ $+\mu \operatorname{diag}\left((1, \ldots, 1)^{\top}\right)_{l \times l} \otimes(0, \ldots, 0, l)_{1 \times l} \otimes(0, \ldots, 0,1)_{(l+1) \times 1}^{\top}$, $\tilde{Q}_{l, l}^{(3,1)}=\sum_{j=1}^{7} \psi^{(j)} Z^{(j, 1)}\left(\S_{1}^{(l, 3)}, \wp_{1}^{(l, 1)}\right) \otimes Z^{(j, 2)}\left(\wp_{2}^{(l, 3)}, \S_{2}^{(l, 1)}\right) \otimes Z^{(j, 3)}\left(\S_{3}^{(l, 3)}, \S_{3}^{(l, 1)}\right)$ $=k_{A} K_{I}\left(0, \ldots, 0, \frac{1}{l-1+K_{I}}\right)_{l \times 1}^{\top} \otimes \operatorname{diag}\left((1, \ldots, 1)^{\top}\right)_{l \times(l+1)}$ $\otimes(0, \ldots, 0, l)_{1 \times(l+1)}$, 


$$
\begin{aligned}
\tilde{Q}_{l, l}^{(3,2)}= & \sum_{j=1}^{7} \psi^{(j)} Z^{(j, 1)}\left(f_{1}^{(l, 3)}, s_{1}^{(l, 2)}\right) \otimes Z^{(j, 2)}\left(f_{2}^{(l, 3)}, f_{2}^{(l, 2)}\right) \otimes Z^{(j, 3)}\left(f_{3}^{(l, 3)}, f_{3}^{(l, 2)}\right) \\
= & k_{B} \operatorname{diag}\left((1, \ldots, 1)^{\top}\right)_{l \times l} \otimes(0, \ldots, 0,1)_{l \times 1}^{\top} \otimes(0, \ldots, 0,1)_{1 \times(l+1)}, \\
\tilde{Q}_{l, l}^{(3,3)}= & \sum_{j=1}^{7} \psi^{(j)} Z^{(j, 1)}\left(f_{1}^{(l, 3)}, f_{1}^{(l, 3)}\right) \otimes Z^{(j, 2)}\left(f_{2}^{(l, 3)}, f_{2}^{(l, 3)}\right) \otimes Z^{(j, 3)}\left(f_{3}^{(l, 3)}, f_{3}^{(l, 3)}\right) \\
= & k_{A} K_{I} \operatorname{superdiag}\left(\left(\frac{1}{K_{I}}, \ldots, \frac{1}{l-2+K_{I}}\right)^{\top}\right)_{l \times l} \otimes \operatorname{diag}\left((1, \ldots, 1)^{\top}\right)_{l \times l} \otimes(l) \\
& +k_{B} \operatorname{diag}\left((1, \ldots, 1)^{\top}\right)_{l \times l} \otimes \operatorname{superdiag}\left((1, \ldots, 1)^{\top}\right)_{l \times l} \otimes(1) \\
& +k_{2} \operatorname{subdiag}\left((1, \ldots, l-1)^{\top}\right)_{l \times l} \otimes \operatorname{subdiag}\left((1, \ldots, l-1)^{\top}\right)_{l \times l} \otimes(1) \\
& +\mu \operatorname{subdiag}\left((1, \ldots, l-1)^{\top}\right)_{l \times l} \otimes \operatorname{diag}\left((1, \ldots, 1)^{\top}\right)_{l \times l} \otimes(1) \\
& +\mu \operatorname{diag}\left((1, \ldots, 1)^{\top}\right)_{l \times l} \otimes \operatorname{subdiag}\left((1, \ldots, l-1)^{\top}\right)_{l \times l} \otimes(1) ;
\end{aligned}
$$

and the nine blocks associated with $Q_{l, l+1}$ are obtained as

$$
\begin{aligned}
& \tilde{Q}_{l, l+1}^{(1,1)}=\sum_{j=1}^{7} \psi^{(j)} Z^{(j, 1)}\left(\oiint_{1}^{(l, 1)}, \S_{1}^{(l+1,1)}\right) \otimes Z^{(j, 2)}\left(\oiint_{2}^{(l, 1)}, \S_{2}^{(l+1,1)}\right) \otimes Z^{(j, 3)}\left(\S_{3}^{(l, 1)}, \S_{3}^{(l+1,1)}\right) \\
& =k_{A} K_{I} \frac{1}{l+K_{I}}(1) \otimes \operatorname{diag}\left((1, \ldots, 1)^{\top}\right)_{(l+1) \times(l+2)} \otimes \operatorname{diag}\left((0, \ldots, l)^{\top}\right)_{(l+1) \times(l+2)}, \\
& \tilde{Q}_{l, l+1}^{(1,2)}=\sum_{j=1}^{7} \psi^{(j)} Z^{(j, 1)}\left(\S_{1}^{(l, 1)}, \S_{1}^{(l+1,2)}\right) \otimes Z^{(j, 2)}\left(\wp_{2}^{(l, 1)}, \wp_{2}^{(l+1,2)}\right) \otimes Z^{(j, 3)}\left(\S_{3}^{(l, 1)}, \wp_{3}^{(l+1,2)}\right) \\
& =k_{B}(0, \ldots, 0,1)_{1 \times(l+1)} \otimes(0, \ldots, 0,1)_{(l+1) \times 1}^{\top} \otimes \operatorname{diag}\left((1, \ldots, 1)^{\top}\right)_{(l+1) \times(l+2)}, \\
& \tilde{Q}_{l, l+1}^{(1,3)}=\sum_{j=1}^{7} \psi^{(j)} Z^{(j, 1)}\left(\S_{1}^{(l, 1)}, \S_{1}^{(l+1,3)}\right) \otimes Z^{(j, 2)}\left(\wp_{2}^{(l, 1)}, \wp_{2}^{(l+1,3)}\right) \otimes Z^{(j, 3)}\left(\S_{3}^{(l, 1)}, \wp_{3}^{(l+1,3)}\right) \\
& =k_{E_{A}} K_{R}\left(0, \ldots, 0, \frac{1}{l+K_{R}}\right)_{1 \times(l+1)} \otimes \operatorname{diag}\left((1, \ldots, 1)^{\top}\right)_{(l+1) \times(l+1)} \\
& \otimes(0, \ldots, 0,1)_{(l+1) \times 1}^{\top}, \\
& \tilde{Q}_{l, l+1}^{(2,1)}=\sum_{j=1}^{7} \psi^{(j)} Z^{(j, 1)}\left(\wp_{1}^{(l, 2)}, \S_{1}^{(l+1,1)}\right) \otimes Z^{(j, 2)}\left(\S_{2}^{(l, 2)}, \wp_{2}^{(l+1,1)}\right) \otimes Z^{(j, 3)}\left(f_{3}^{(l, 2)}, \wp_{3}^{(l+1,1)}\right) \\
& =0_{l(l+1) \times(l+2)^{2}}, \\
& \tilde{Q}_{l, l+1}^{(2,2)}=\sum_{j=1}^{7} \psi^{(j)} Z^{(j, 1)}\left(\wp_{1}^{(l, 2)}, \S_{1}^{(l+1,2)}\right) \otimes Z^{(j, 2)}\left(\wp_{2}^{(l, 2)}, \wp_{2}^{(l+1,2)}\right) \otimes Z^{(j, 3)}\left(f_{3}^{(l, 2)}, \S_{3}^{(l+1,2)}\right) \\
& =k_{B} \operatorname{diag}\left((1, \ldots, 1)^{\top}\right)_{l \times(l+1)} \otimes(1) \otimes \operatorname{diag}\left((1, \ldots, 1)^{\top}\right)_{(l+1) \times(l+2)},
\end{aligned}
$$

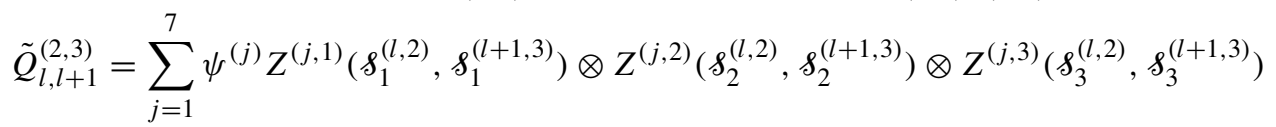

$$
\begin{aligned}
& =k_{E_{A}} K_{R} \operatorname{diag}\left(\left(\frac{1}{K_{R}}, \ldots, \frac{1}{l-1+K_{R}}\right)^{\top}\right)_{l \times(l+1)} \otimes(0, \ldots, 0,1)_{1 \times(l+1)} \\
& \otimes(0, \ldots, 0,1)_{(l+1) \times 1}^{\top} \text {, }
\end{aligned}
$$




$$
\begin{aligned}
& \tilde{Q}_{l, l+1}^{(3,1)}=\sum_{j=1}^{7} \psi^{(j)} Z^{(j, 1)}\left(\S_{1}^{(l, 3)}, \S_{1}^{(l+1,1)}\right) \otimes Z^{(j, 2)}\left(\wp_{2}^{(l, 3)}, \S_{2}^{(l+1,1)}\right) \otimes Z^{(j, 3)}\left(f_{3}^{(l, 3)}, \S_{3}^{(l+1,1)}\right) \\
& =0_{l^{2} \times(l+2)^{2}}, \\
& \tilde{Q}_{l, l+1}^{(3,2)}=\sum_{j=1}^{7} \psi^{(j)} Z^{(j, 1)}\left(\S_{1}^{(l, 3)}, \S_{1}^{(l+1,2)}\right) \otimes Z^{(j, 2)}\left(\S_{2}^{(l, 3)}, \S_{2}^{(l+1,2)}\right) \otimes Z^{(j, 3)}\left(\S_{3}^{(l, 3)}, \S_{3}^{(l+1,2)}\right) \\
& =0_{l^{2} \times(l+1)(l+2)}, \\
& \tilde{Q}_{l, l+1}^{(3,3)}=\sum_{j=1}^{7} \psi^{(j)} Z^{(j, 1)}\left(\S_{1}^{(l, 3)}, \S_{1}^{(l+1,3)}\right) \otimes Z^{(j, 2)}\left(\S_{2}^{(l, 3)}, \S_{2}^{(l+1,3)}\right) \otimes Z^{(j, 3)}\left(\S_{3}^{(l, 3)}, \S_{3}^{(l+1,3)}\right) \\
& =k_{E_{A}} K_{R} \operatorname{diag}\left(\left(\frac{1}{K_{R}}, \ldots, \frac{1}{l-1+K_{R}}\right)^{\top}\right)_{l \times(l+1)} \otimes \operatorname{diag}\left((1, \ldots, 1)^{\top}\right)_{l \times(l+1)} \otimes(1) .
\end{aligned}
$$

\section{Acknowledgement}

The work of the second author is supported by The Scientific and Technological Research Council of Turkey.

\section{References}

[1] Baumann, H. and Sandmann, W. (2010). Numerical solution of level dependent quasi-birth-and-death processes. Procedia Comput. Sci. 1, 1561-1569.

[2] BRight, L. AND TAYlor, P. G. (1995). Calculating the equilibrium distribution in level dependent quasi-birthand-death processes. Commun. Statist. Stoch. Models 11, 497-525.

[3] Buchiolz, P. (1994). A class of hierarchical queueing networks and their analysis. Queueing Systems 15, 59-80.

[4] Dayar, T. (2006). Analyzing Markov chains based on Kronecker products. In Markov Anniversary Meeting, eds A. N. Langville and W. J. Stewart, Boson Books, Raleigh, NC, pp. 279-300.

[5] DaYAR, T. And ORHAN, M. C. (2011). LDQBD solver version 2. Available at http://www.cs.bilkent.edu.tr/tugrul/ software.html.

[6] Dayar, T., Hermanns, H., Spieler, D. And Wolf, V. (2011). Bounding the equilibrium distribution of Markov population models. Numer. Linear Algebra Appl. 18, 931-946.

[7] Dayar, T., Sandmann, W., Spieler, D. and Wolf, V. (2011). Infinite level-dependent QBD processes and matrix analytic solutions for stochastic chemical kinetics. Adv. Appl. Prob. 43, 1005-1026.

[8] Gans, D. (1969). Transformations and Geometries. Appleton-Century-Crofts, New York.

[9] Gillespie, D. T. (1977). Exact stochastic simulation of coupled chemical reactions. J. Phys. Chem. 81, 23402361.

[10] Glynn, P. W. AND Zeevi, A. (2008). Bounding stationary expectations of Markov processes. In Markov Processes and Related Topics (Inst. Math. Statist. Collect. 4), Institute of Mathematical Statistics, Beachwood, OH, pp. 195-214.

[11] Grassman, W. K. (ed.) (2000). Computational Probability. Kluwer, Norwell, MA.

[12] Grassman, W. K. and Stanford, D. (2000). Matrix analytic methods. In Computational Probability, ed. W. K. Grassman, Kluwer, Norwell, MA, pp. 153-204.

[13] Hanschke, T. (1999). A matrix continued fraction algorithm for the multiserver repeated order queue. Math. Comput. Modelling 30, 159-170.

[14] Kurtz, T. G. (1972). The relationship between stochastic and deterministic models for chemical reactions. J. Chem. Phys. 57, 2976-2978.

[15] Latouche, G. and Ramaswami, V. (1999). Introduction to Matrix Analytic Methods in Stochastic Modeling. SIAM, Philadelphia, PA.

[16] LEE, T. L., LI, T. Y. AND TsAI, C. H. (2008). HOM4PS-2.0: a software package for solving polynomial systems by the polyhedral homotopy continuation method. Computing 83, 109-133.

[17] Li, H., Cao, Y., Petzold, L. R. And Gillespie, D. T. (2008). Algorithms and software for stochastic simulation of biochemical reacting systems. Biotech. Progress 24, 56-61.

[18] Loinger, A. And Biham, O. (2007). Stochastic simulations of the repressilator circuit. Phys. Rev. E 76, 051917. 
[19] McQuarrie, D. A. (1967). Stochastic approach to chemical kinetics. J. Appl. Prob. 4, 413-478.

[20] Neuts, M. F. (1981). Matrix-Geometric Solutions in Stochastic Models. Johns Hopkins University Press, Baltimore, MD.

[21] ORHAN, M. C. (2011). Kronecker-based infinite level-dependent QBDs: matrix analytic solution versus simulation. Masters Thesis, Department of Computer Engineering, Bilkent University.

[22] Plateau, B. (1985). On the stochastic structure of parallelism and synchronization models for distributed algorithms. In Proc. ACM SIGMETRICS 1985 (Austin, Texas), ACM, New York, pp. 147-154.

[23] Plateau, B. and Stewart, W. J. (2000). Stochastic automata networks. In Computational Probability, ed. W. Grassmann, Kluwer, Norwell, MA, pp. 113-152.

[24] Pollett, P. K. and Taylor, P. G. (1993). On the problem of establishing the existence of stationary distributions for continuous-time Markov chains. Prob. Eng. Inf. Sci. 7, 529-543.

[25] Ramaswami, V. And TaYlor, P. G. (1996). Some properties of the rate operators in level dependent quasi-birthand-death processes with a countable number of phases. Commun. Statist. Stoch. Models 12, 143-164.

[26] SANFT, K. R. et al. (2011). Stochkit2: software for discrete stochastic simulation of biochemical systems with events. Bioinformatics 11, 2457-2458.

[27] Singer, K. (1953). Application of the theory of stochastic processes to the study of irreproducible chemical reactions and nucleation processes. J. R. Statist. Soc. B 15, 92-106.

[28] SJöberg, P. L., Lötstedt, P. ANd Elf, J. (2009). Fokker-Planck approximation of the master equation in molecular biology. Comput. Visualization Science 12, 37-50.

[29] Stein, S. K. And Barcellos, A. (1992). Calculus and Analytic Geometry. McGraw-Hill, New York.

[30] Stewart, W. J. (1994). Introduction to the Numerical Solution of Markov Chains. Princeton University Press, Princeton, NJ.

[31] Thattai, M. and van Oudenaarden, A. (2001). Intrinsic noise in gene regulatory networks. Proc. Nat. Acad. Sci. USA 98, 8614-8619.

[32] Tweedie, R. L. (1975). Sufficient conditions for regularity, recurrence and ergodicity of Markov processes. Math. Proc. Camb. Phil. Soc. 78, 125-136.

[33] Van Kampen, N. G. (1992). Stochastic Processes in Physics and Chemistry. North-Holland, Amsterdam. 